\title{
THE DESCRIPTIVE SET-THEORETIC COMPLEXITY OF THE SET OF POINTS OF CONTINUITY OF A MULTI-VALUED FUNCTION*
}

\author{
VASSILIOS GREGORIADES
}

Fachbereich Mathematik, Technische Universität Darmstadt, Schlossgartenstr. 7, 64289 Darmstadt, Germany

e-mail address: gregoriades [at] mathematik [dot] tu-darmstadt [dot] de

\begin{abstract}
In this article we treat a notion of continuity for a multi-valued function $F$ and we compute the descriptive set-theoretic complexity of the set of all $x$ for which $F$ is continuous at $x$. We give conditions under which the latter set is either a $G_{\delta}$ set or the countable union of $G_{\delta}$ sets. Also we provide a counterexample which shows that the latter result is optimum under the same conditions. Moreover we prove that those conditions are necessary in order to obtain that the set of points of continuity of $F$ is Borel i.e., we show that if we drop some of the previous conditions then there is a multi-valued function $F$ whose graph is a Borel set and the set of points of continuity of $F$ is not a Borel set. Finally we give some analogous results regarding a stronger notion of continuity for a multi-valued function. This article is motivated by a question of Martin Ziegler (TU Darmstadt).
\end{abstract}

\section{INTRODUCTION.}

A multi-valued function $F$ from a set $X$ to another set $Y$ is any function from $X$ to the power set of $Y$ i.e., $F$ assigns sets to points. Such a function will be denoted by $F: X \Rightarrow Y$. A multi-valued function $F: X \Rightarrow Y$ can be identified with its graph $G r(F) \subseteq X \times Y$ which is defined by

$$
(x, y) \in G r(F) \Longleftrightarrow y \in F(x) .
$$

This way we view $F$ as a subset of $X \times Y$. From now on we assume that all given multivalued functions are between metric spaces and that they are total i.e., if $F: X \Rightarrow Y$ is

1998 ACM Subject Classification: F.4.1.

Key words and phrases: Multi-valued function, points of continuity, $\Sigma_{n}^{0}$ set, Borel set, analytic set, descriptive set theory.

* An extended abstract of this article has been published in the proceedings of the $7^{\text {th }}$ International Conference on Computability and Complexity in Analysis.

The author is currently a post-doctoral researcher at the Mathematics Department of TU Darmstadt in the work-group of ULRICH KoHLENBACH (TU Darmstadt), whom the author would like to thank. The author owns special thanks to MARTIN ZIEGLER (TU Darmstadt) for bringing to the author's attention the problem which motivated this article and for his valuable advice. Finally the author would like to thank all referees of this article (including the ones of the submission to the proceedings of the $7^{\text {th }}$ International Conference on Computability and Complexity in Analysis) for their kind remarks, comments and suggestions. 
given then $F(x) \neq \emptyset$ for all $x \in X$, in other words the projection of $F$ along $Y$ is the whole space $X$.

There are various notions of continuity for multi-valued functions, here we focus on two of those (see [2] Definition 2.1, 3] pp. 70-71 and [1] p. 82, p. 93).

Definition 1.1. Let $(X, p)$ and $(Y, d)$ be metric spaces; a multi-valued function $F: X \Rightarrow Y$ is continuous at $x$ if there is some $y \in F(x)$ such that for all $\varepsilon>0$ there is some $\delta>0$ such that for all $x^{\prime} \in B_{p}(x, \delta)$ there is some $y^{\prime} \in F\left(x^{\prime}\right)$ for which we have that $d\left(y, y^{\prime}\right)<\varepsilon$.

Definition 1.2. Let $(X, p)$ and $(Y, d)$ be metric spaces; a multi-valued function $F: X \Rightarrow Y$ is strongly continuous at $x$ if for all $y \in F(x)$ and for all $\varepsilon>0$ there is some $\delta>0$ such that for all $x^{\prime} \in B_{p}(x, \delta)$ there is some $y^{\prime} \in F\left(x^{\prime}\right)$ for which we have that $d\left(y, y^{\prime}\right)<\varepsilon$.

It is clear that both these notions generalize the classical notion of continuity of functions. Moreover it is also clear that the continuity/strong continuity of a multi-valued function is preserved under distance functions which generate the same topology.

The motivation of this article is the following question posed by M. Ziegler in [6] (Question 63a)). It is well known that if we have a function $f:(X, p) \rightarrow(Y, d)$ then the set of points of continuity of $f$ is a $G_{\delta}$ subset of $X$; see for example 3.B in [4. So what can be said about the descriptive set-theoretic complexity of the set of points of continuity/strong continuity of a multi-valued function $F:(X, p) \Rightarrow(Y, d)$ ? In this article we present the answer for the case of continuity and present some analogous results for the case of strong continuity. The full answer for the latter case is still under investigation.

We proceed with basic terminology and notations. By $\omega$ we denote the set of natural numbers (including the number 0). Suppose that $X$ and $Y$ are two topological spaces. We call a function $f: X \rightarrow Y$ a topological isomorphism between $X$ and $Y$ if the function $f$ is bijective, continuous and the function $f^{-1}$ is continuous. We will also say that the space $X$ is topologically isomorphic with $Y$ if there exists a topological isomorphism between $X$ and $Y$.

The Baire space $\mathcal{N}$ is the set of all sequences of naturals i.e., $\mathcal{N}=\omega^{\omega}$ with the usual product topology. We call the members of the Baire space fractions and we usually denote them by lower case Greek letters $\alpha, \beta$ etc. One choice of basic neighborhoods for the product topology on $\mathcal{N}$ is the collection of the following sets

$$
N\left(k_{0}, \ldots, k_{n-1}\right)=\left\{\alpha \in \mathcal{N} \mid \alpha(0)=k_{0}, \ldots, \alpha(n-1)=k_{n-1}\right\}
$$

where $k_{0}, \ldots, k_{n-1} \in \omega$. The set of ultimately constant sequences is clearly countable and dense in $\mathcal{N}$; thus the latter is a separable space. For $\alpha, \beta \in \mathcal{N}$ with $\alpha \neq \beta$ define

$$
d_{\mathcal{N}}(\alpha, \beta)=1 /(\text { least } n[\alpha(n) \neq \beta(n)]+1) .
$$

Also put $d_{\mathcal{N}}(\alpha, \alpha)=0$ for all $\alpha \in \mathcal{N}$. It is not hard to see that the function $d_{\mathcal{N}}$ is a complete distance function on $\mathcal{N}$ which generates its topology. From now on we think of the Baire space $\mathcal{N}$ with this distance function $d_{\mathcal{N}}$.

We denote by $\mathcal{C}$ the subset of the Baire space $\mathcal{N}$ which consist of all sequences with values 0 and 1 i.e., $\mathcal{C}=2^{\omega}$. The set $\mathcal{C}$ with the induced topology is a compact space. It is not hard to see that $\mathcal{C}$ is topologically isomorphic with the usual Cantor set of the unit interval. This result motivates us to call $\mathcal{C}$ Cantor space.

We denote by $\omega^{\star}$ the set of all finite sequences of $\omega$. If $u \in \omega^{\star}$ then there are unique naturals $n, k_{0}, \ldots, k_{n-1}$ such that $u=\left(k_{0}, \ldots, k_{n-1}\right)$. The length of $u$ is the previous natural $n$ and we denote it by $\operatorname{lh}(u)$. Also we write $u(i)=k_{i}$ for all $i<l h(u)$, so that 
$u=(u(0), \ldots, u(\operatorname{lh}(u)-1))$. It is convenient to include the empty sequence in $\omega^{\star}$ i.e., the one with zero length. The latter will be denoted by $\langle\cdot\rangle$. So when we write $u=(u(0), \ldots, u(n-1))$ we will always mean in case where $n=0$ that $u=\langle\cdot\rangle$. If $u \in \omega^{\star}$ and $n \in \omega$ we denote the finite sequence $(u(0), \ldots, u(\operatorname{lh}(u)-1), n)$ by $u^{\wedge}(n)$. We write $u \sqsubseteq v$ exactly when $\operatorname{lh}(u) \leq \operatorname{lh}(v)$ and $u(i)=v(i)$ for all $i<\operatorname{lh}(u)$ i.e., $u \sqsubseteq v$ means that $v$ is an extension of $u$ or equivalently $u$ is an initial segment of $v$.

A set $T \subseteq \omega^{\star}$ is called a tree on $\omega$ if it is closed under initial segments i.e.,

$$
v \in T \& u \sqsubseteq v \Longrightarrow u \in T \text {. }
$$

The members of a tree $T$ are called nodes or branches of $T$. A tree $T$ is of finite branching if and only if for all $u \in T$ there are only finitely many $n \in \omega$ such that $u^{\wedge}(n) \in T$. A fraction $\alpha$ is an infinite branch of $T$ if and only if for all $n \in \omega$ we have that $(\alpha(0), \ldots, \alpha(n-1)) \in T$. The body $[T]$ of a tree $T$ is the set of infinite branches of $T$.

For practical reasons when we refer to a tree $T$ we will always assume that $T$ is not empty i.e., $\langle\cdot\rangle \in T$. Define

$$
\operatorname{Tr}=\left\{T \subseteq \omega^{\star} \mid \text { the set } T \text { is a tree on } \omega\right\} .
$$

We may view every $T \in T r$ as a member of $2^{\omega^{\star}}$ by identifying $T$ with its characteristic function $\chi_{T}: \omega^{\star} \rightarrow\{0,1\}$. Since the set $\omega^{\star}$ is countable the space $2^{\omega^{\star}}$ with the product topology is completely metrizable - in fact it is topologically isomorphic with the Cantor space $\mathcal{C}$. Moreover the set $\operatorname{Tr}$ is a closed subset of $\mathcal{C}$. Indeed let $T_{i} \in \operatorname{Tr}$ for all $i \in \omega$ be such that $T_{i} \stackrel{i \rightarrow \infty}{\longrightarrow} S$ for some $S \in 2^{\omega^{\star}}$; we will prove that $S \in T r$. From the hypothesis it follows that for all $u \in \omega^{\star}$ there is some $i_{0} \in \omega$ such that for all $i \geq i_{0}$ we have that

$$
u \in T_{i} \Longleftrightarrow u \in S \text {. }
$$

Taking $u=\langle\cdot\rangle$ since $T_{i} \in \operatorname{Tr}$ for all $i \in \omega$ we have that $\langle\cdot\rangle \in S$ and so $S$ is not empty. Also if $u, v \in \omega^{\star}$ we find $i$ large enough so that $u \in T_{i} \Longleftrightarrow u \in S$ and $v \in T_{i} \Longleftrightarrow v \in S$. So if $u \in S$ and $v \sqsubseteq u$ then $u \in T_{i}$ and since $T_{i}$ is a tree we also have that $v \in T_{i}$; hence $v \in S$. Therefore $S \in \operatorname{Tr}$ and the set of trees $\operatorname{Tr}$ is closed in $\mathcal{C}$.

We make a final comment about trees. For any non-empty set $S$ of finite sequences of naturals the tree $T$ which is generated by $S$ is the following

$$
\{u \mid(\exists w \in S)[u \sqsubseteq w]\}
$$

i.e., the tree which is generated by $S$ is the tree which arises by taking all initial segments of members of $S$.

Suppose that $X$ is a metric space. The family $\Sigma_{1}^{0}(X)$ is the collection of all open subsets of $X$. Inductively we define the family $\Sigma_{n+1}^{0}(X)$ for $n \geq 1$ as follows: for $A \subseteq X$,

$$
A \in \Sigma_{n+1}^{0}(X) \Longleftrightarrow A=\bigcup_{i \in \omega} A_{i}, \quad \text { where } X \backslash A_{i} \in \Sigma_{k_{i}}^{0}(X) \text { for some } k_{i} \leq n \text { for all } i \in \omega .
$$

Put also

$$
\Pi_{n}^{0}(X)=\left\{B \subseteq X \quad \mid X \backslash B \in \Sigma_{n}^{0}(X)\right\}
$$

and $\Delta_{n}^{0}(X)=\Sigma_{n}^{0}(X) \cap \Pi_{n}^{0}(X)$ for all $n \geq 1$. Notice that family $\Pi_{1}^{0}(X)$ is the collection of all closed subsets of $X$, the family $\Sigma_{2}^{0}(X)$ is the collection of all $F_{\sigma}$ subsets of $X$ and so on. By a simple induction one can prove that $\Sigma_{n}^{0}(X) \cup \Pi_{n}^{0}(X) \subseteq \Delta_{n+1}^{0}(X)$ for all $n \geq 1$. It is well known that in case where $X$ admits a complete distance function and it is an uncountable set then $\Sigma_{n}^{0}(X) \neq \Pi_{n}^{0}(X)$ for all $n \geq 1$ and so the previous inclusion is a proper one for all $n \geq 1$, (see [4] and [5]). 
The families $\Sigma_{n}^{0}(X), \Pi_{n}^{0}(X)$ are closed under finite unions, finite intersections, and continuous pre-images i.e., if $f: X \rightarrow Y$ is continuous and $B \subseteq Y$ is in $\Sigma_{n}^{0}(Y)$ then $f^{-1}[B]$ is in $\Sigma_{n}^{0}(X)$. Moreover it is clear that if $f: X \rightarrow Y$ is a topological isomorphism then for all $A \subseteq X$ we have that $A \in \Sigma_{n}^{0}(X)$ if and only if $f[A] \in \Sigma_{n}^{0}(Y)$ and similarly for $\Pi_{n}^{0}$ for all $n \in \omega$. Finally the family is $\Sigma_{n}^{0}(X)$ is closed under countable unions, the family $\Pi_{n}^{0}(X)$ is closed under countable intersections and the family $\Delta_{n}^{0}(X)$ is closed under complements. We usually say that $A$ is in $\Sigma_{n}^{0}$ when $X$ is easily understood from the context. It is clear that all sets in $\Sigma_{n}^{0}$ are Borel sets.

We now deal with a bigger family of sets. Suppose that $X$ is separable and that $X$ admits a complete distance function. A set $A \subseteq X$ is in $\Sigma_{1}^{1}(X)$ or it is analytic if $A$ is the continuous image of a closed subset of a complete and separable metric space $1 \frac{1}{1}$ It is well known that in the definition of analytic sets we may replace the term "continuous image" by "Borel image" (i.e., image under a Borel measurable function) and/or the term "closed subset" by "Borel set", (see [4] and [5]). A set $B \subseteq X$ is in $\Pi_{1}^{1}(X)$ or it is co-analytic if the set $X \backslash B$ is analytic and $B$ is in $\Delta_{1}^{1}(X)$ or it is bi-analytic if $B$ is both analytic and co-analytic. It is well known that every Borel set is analytic, hence every Borel set is bi-analytic. A classical theorem states that a set $B$ is Borel if and only if it is bi-analytic, (see [5] 2E.1 and 2E.2).

The families $\Sigma_{1}^{1}(X), \Pi_{1}^{1}(X)$ and $\Delta_{1}^{1}(X)$ are closed under countable unions, countable intersections and continuous pre-images. Moreover the family $\Sigma_{1}^{1}(X)$ is closed under Borel images i.e., if $Y$ is a complete and separable metric space, $f: X \rightarrow Y$ is a Borel measurable function and $A$ is an analytic subset of $X$ then $f[A]$ is an analytic subset of $Y$. Finally if $X$ is uncountable we have that $\Sigma_{1}^{1}(X) \neq \Pi_{1}^{1}(X)$ and in particular there is an analytic set which is not Borel.

We can pursue this hierarchy further by defining the family $\Sigma_{n+1}^{1}(X)$ as the collection of all subsets of $X$ which are the continuous image of a $\Pi_{n}^{1}$ subset of a complete and separable metric space. Similarly one defines the family $\Pi_{n+1}^{1}(X)$ as the collection of all subsets of $X$ whose complement is in $\Sigma_{n+1}^{1}(X)$ and the family $\Delta_{n+1}^{1}(X)$ as the collection of all subsets of $X$ which belong both to $\Sigma_{n+1}^{1}(X)$ and $\Pi_{n+1}^{1}(X)$. By a simple induction one can prove that $\Sigma_{n}^{1}(X) \cup \Pi_{n}^{1}(X) \subseteq \Delta_{n+1}^{1}(X)$ for all $n \geq 1$. Also the analogous properties stated above are true. The reader may refer to [5] for more information on those classes.

The proofs of the forthcoming theorems make a substantial use of techniques of Descriptive Set Theory which involve the use of many quantifiers. Of course those quantifiers can be interpreted as unions and intersections of sets and this is what we usually do in order to prove that a given set is for example $\Pi_{2}^{0}$. There are some cases though (for example in the proof of Theorem 2.6) where this interpretation becomes too complicated. In these cases it is better to think of a given set $P$ as a relation in order to derive its complexity. The reader can consult section $1 \mathrm{C}$ of [5] on how one can make computations with relations.

\section{Results about the Set of points of CONtinuity of A Multi-VAlued Function.}

In this section we examine the set of points of continuity (as given in Definition 1.1) of a multi-valued function. We can classify our results by two groups: the "low-level" group where the set of points of continuity is at most a $\Sigma_{3}^{0}$ set and the "higher level group" where

\footnotetext{
${ }^{1}$ The notion of an analytic set can be treated in a more general context of spaces; however we prefer to stay in the context of complete and separable metric spaces.
} 
the set of points of continuity is not even a Borel set, while on the other hand under some reasonable assumptions it is an analytic set.

The case of low-level classification in the Borel hierarchy. We begin with some positive results regarding the set of points of continuity of a multi-valued function $F$. Recall that a topological space $Y$ is exhaustible by compact sets if there is a sequence $\left(K_{n}\right)_{n \in \omega}$ of compact subsets of $Y$ such that every $K_{n}$ is contained in the interior of $K_{n+1}$ and $Y=\bigcup_{n \in \omega} K_{n}$. Notice the lack of any hypothesis about the set $F$ in the next theorem.

Theorem 2.1. Let $(X, p)$ and $(Y, d)$ be metric spaces with $(Y, d)$ being separable and let $F: X \Rightarrow Y$ be a multi-valued function.

(a) If the set $F(x)$ is compact for all $x \in X$ then the set of points of continuity of $F$ is $\Pi_{2}^{0}$ i.e., $G_{\delta}$.

(b) If $Y$ is exhaustible by compact sets and the set $F(x)$ is closed for all $x \in X$, then the set of points of continuity of $F$ is $\Sigma_{3}^{0}$.

Proof. For $y \in Y$ and for a non-empty $A \subseteq Y$ we denote by $d(y, A)$ the non-negative number $\inf \{d(y, z) \mid z \in A\}$. It is clear that when $A$ is compact then $d(y, A)=d\left(y, z_{0}\right)$ for some $z_{0} \in A$. It is useful to adopt the notation $d(y, \emptyset)=1$. We denote by $P$ the set of $x$ 's for which $F$ is continuous at $x$ and also we fix a sequence $\left(y_{s}\right)_{s \in \omega}$ in $Y$ for which the set $\left\{y_{s} \mid s \in \omega\right\}$ is dense in $Y$.

We begin with assertion (a). We claim that

$$
x \in P \Longleftrightarrow(\forall n)(\exists s) \inf \left\{\sup \left\{d\left(y_{s}, F\left(x^{\prime}\right)\right) \mid x^{\prime} \in B_{p}(x, \delta)\right\} \quad \mid \delta>0\right\}<\frac{1}{n+1}
$$

Assume that $x \in P$ and let $n \in \omega$. There exists $y \in F(x)$ such that for all $\varepsilon>0$ there is some $\delta>0$ such that for all $x^{\prime} \in B_{p}(x, \delta)$ there is some $y^{\prime} \in F\left(x^{\prime}\right)$ for which we have that $d\left(y, y^{\prime}\right)<\varepsilon$. Choose $s \in \omega$ such that

$$
d\left(y, y_{s}\right)<\frac{1}{2(n+1)}
$$

We will show that $\inf \left\{\sup \left\{d\left(y_{s}, F\left(x^{\prime}\right)\right) \quad \mid x^{\prime} \in B_{p}(x, \delta)\right\} \quad \mid \delta>0\right\}<\frac{1}{n+1}$. For this it is enough to find some $\delta>0$ for which we have that

$$
\sup \left\{d\left(y_{s}, F\left(x^{\prime}\right)\right) \mid x^{\prime} \in B_{p}(x, \delta)\right\}<\frac{1}{n+1}
$$

Let $\varepsilon=\frac{1}{2(n+1)}-d\left(y, y_{s}\right)>0$ and choose $\delta>0$ such that for all $x^{\prime} \in B_{p}(x, \delta)$ there is some $y^{\prime} \in F\left(x^{\prime}\right)$ for which we have that $d\left(y, y^{\prime}\right)<\varepsilon$. We claim that this $\delta$ satisfies (1). Indeed for $x^{\prime} \in B_{p}(x, \delta)$ choose a $y^{\prime} \in F\left(x^{\prime}\right)$ which satisfies $d\left(y, y^{\prime}\right)<\varepsilon$ and compute

$$
d\left(y_{s}, F\left(x^{\prime}\right)\right) \leq d\left(y_{s}, y^{\prime}\right) \leq d\left(y_{s}, y\right)+d\left(y, y^{\prime}\right)<d\left(y_{s}, y\right)+\varepsilon=\frac{1}{2(n+1)} .
$$

So $\sup \left\{d\left(y_{s}, F\left(x^{\prime}\right)\right) \quad \mid x^{\prime} \in B_{p}(x, \delta)\right\} \leq \frac{1}{2(n+1)}<\frac{1}{n+1}$ and we have proved the left-to-righthand direction.

Now we deal with the inverse direction. Assume the right-hand condition in $(*)$. It follows that there exists a sequence $\left(s_{n}\right)_{n \in \omega}$ of naturals such that

$$
\inf \left\{\sup \left\{d\left(y_{s_{n}}, F\left(x^{\prime}\right)\right) \mid x^{\prime} \in B_{p}(x, \delta)\right\} \quad \mid \delta>0\right\}<\frac{1}{n+1}
$$


for all $n \in \omega$. So for all $n \in \omega$ there is some $\delta_{n}>0$ such that

$$
\sup \left\{d\left(y_{s_{n}}, F\left(x^{\prime}\right)\right) \mid x^{\prime} \in B_{p}\left(x, \delta_{n}\right)\right\}<\frac{1}{n+1} .
$$

In particular we have that $d\left(y_{s_{n}}, F(x)\right)<\frac{1}{n+1}$ for all $n \in \omega$. There is some $z_{n} \in F(x)$ such that $d\left(y_{s_{n}}, z_{n}\right)<\frac{1}{n+1}$, for all $n \in \omega$. From the compactness of $F(x)$ there is a subsequence $\left(z_{k_{n}}\right)_{n \in \omega}$ of $\left(z_{n}\right)_{n \in \omega}$ which converges to some $y \in F(x)$. And since $d\left(y_{s_{n}}, z_{n}\right)<\frac{1}{n+1}$ for all $n \in \omega$ we also have that the sequence $\left(y_{s_{k_{n}}}\right)_{n \in \omega}$ is convergent to $y$.

We claim that this $y \in F(x)$ witnesses that $x \in P$. Suppose that we are given $\varepsilon>0$. Since $y_{s_{k_{n}}} \stackrel{n \in \omega}{\longrightarrow} y$ there is some $n_{0}$ such that for all $n \geq n_{0}$ we have that

$$
d\left(y_{s_{n}}, y\right)<\frac{\varepsilon}{2}
$$

Choose some $n_{1} \geq n_{0}$ for which we have that $\frac{1}{n_{1}}<\frac{\varepsilon}{2}$. Applying (2) to the natural $k_{n_{1}}$ we obtain that there is some $\delta>0$ such that

$$
\sup \left\{d\left(y_{s_{k_{1}}}, F\left(x^{\prime}\right)\right) \mid x^{\prime} \in B_{p}(x, \delta)\right\}<\frac{1}{k_{n_{1}}+1} .
$$

Let $x^{\prime} \in B_{p}(x, \delta)$; since $F\left(x^{\prime}\right)$ is compact there is some $y^{\prime} \in F\left(x^{\prime}\right)$ such that $d\left(y_{s_{k_{n}}}, F\left(x^{\prime}\right)\right)=$ $d\left(y_{s_{k_{1}}}, y^{\prime}\right)$. We will prove that $d\left(y, y^{\prime}\right)<\varepsilon$. Indeed

$$
\begin{aligned}
d\left(y, y^{\prime}\right) & \leq d\left(y, y_{s_{k_{1}}}\right)+d\left(y_{s_{k_{n}}}, y^{\prime}\right) \\
& <\frac{\varepsilon}{2}+d\left(y_{s_{k_{1}}}, F\left(x^{\prime}\right)\right) \\
& <\frac{\varepsilon}{2}+\frac{1}{k_{n_{1}}+1}<\frac{\varepsilon}{2}+\frac{1}{n_{1}}<\varepsilon .
\end{aligned}
$$

Thus we have proved $(*)$. Now for fixed $n, s \in \omega$ we define the set $Q_{n, s}$ as follows:

$$
x \in Q_{n, s} \Longleftrightarrow \inf \left\{\sup \left\{d\left(y_{s}, F\left(x^{\prime}\right)\right) \mid x^{\prime} \in B_{p}(x, \delta)\right\} \mid \delta>0\right\}<\frac{1}{n+1},
$$

so that from $(*)$ we have that $P=\bigcap_{n} \bigcup_{s} Q_{n, s}$. The proof will be complete as long as we prove that the set $Q_{n, s}$ is open for all $n, s$. The latter assertion is immediate from the fact that the ball $B_{p}(x, \delta)$ is open. To see this fix $n, s \in \omega$ and let $x \in Q_{n, s}$. Then there is some $\delta>0$ such that

$$
\sup \left\{d\left(y_{s}, F\left(x^{\prime}\right)\right) \mid x^{\prime} \in B_{p}(x, \delta)\right\}<\frac{1}{n+1} .
$$

We claim that $B_{p}(x, \delta) \subseteq Q_{n, s}$. Indeed let $w \in B_{p}(x, \delta)$; since the latter set is open there is some $\delta_{w}>0$ such that $B_{p}\left(w, \delta_{w}\right) \subseteq B_{p}(x, \delta)$. Hence

$$
\left\{d\left(y_{s}, F\left(x^{\prime}\right)\right) \mid x^{\prime} \in B_{p}\left(w, \delta_{w}\right)\right\} \subseteq\left\{d\left(y_{s}, F\left(x^{\prime}\right)\right) \mid x^{\prime} \in B_{p}(x, \delta)\right\}
$$

and so

$$
\sup \left\{d\left(y_{s}, F\left(x^{\prime}\right)\right) \mid x^{\prime} \in B_{p}\left(w, \delta_{w}\right)\right\} \leq \sup \left\{d\left(y_{s}, F\left(x^{\prime}\right)\right) \quad \mid x^{\prime} \in B_{p}(x, \delta)\right\}<\frac{1}{n+1} .
$$


Therefore

$$
\begin{aligned}
& \inf \left\{\sup \left\{d\left(y_{s}, F\left(x^{\prime}\right)\right) \mid x^{\prime} \in B_{p}(w, \delta)\right\} \mid \delta>0\right\} \\
\leq & \sup \left\{d\left(y_{s}, F\left(x^{\prime}\right)\right) \mid x^{\prime} \in B_{p}\left(w, \delta_{w}\right)\right\} \\
< & \frac{1}{n+1}
\end{aligned}
$$

i.e., $w \in Q_{n, s}$ and we are done.

The following remark is motivated by the comments of one of the referees of this article, who noticed that we can eliminate one instance of our hypothesis about compactness. The author would like to express his thanks.

Remark. The reader may notice that the only place in the whole proof of (a) where the hypothesis about compactness is needed is in the inverse direction of $(*)$. The use of compactness of $F\left(x^{\prime}\right)$ for $x^{\prime} \in B_{p}(x, \delta)$ can be avoided by choosing $y^{\prime} \in F\left(x^{\prime}\right)$ such that $d\left(y_{s_{k_{1}}}, y^{\prime}\right)<d\left(y_{s_{k_{1}}}, F\left(x^{\prime}\right)\right)+\frac{\varepsilon}{3}$ and by replacing the previous occurrences of $\frac{\varepsilon}{2}$ by $\frac{\varepsilon}{3}$. In conclusion what is proved here is the slightly stronger result: if $Y$ is separable, $x \in X$ and $F(x)$ is compact, then $F$ is continuous at $x$ exactly when the right hand of $(*)$ holds.

We now prove (b). Let $\left(K_{m}\right)_{m \in \omega}$ be a sequence of compact subsets of $Y$ such that $K_{m} \subseteq K_{m+1}^{\circ}$ for all $m \in \omega$ and $Y=\bigcup_{m \in \omega} K_{m}$. We claim the following modified version of $(*)$ :

$$
\begin{aligned}
x \in P \Longleftrightarrow & (\exists m)(\forall n)(\exists s) \\
& \inf \left\{\sup \left\{d\left(y_{s}, F\left(x^{\prime}\right) \cap K_{m}\right) \mid x^{\prime} \in B_{p}(x, \delta)\right\} \quad \mid \delta>0\right\}<\frac{1}{n+1}
\end{aligned}
$$

The proof of $(\dagger)$ is similar to the proof of $(*)$; however some mild changes are needed. We give a sketch of this proof. For the left-to-right-hand direction let $y \in Y$ which witnesses that $x$ is a point of continuity of $F$. Let $m$ be such that $y \in K_{m}^{\circ}$ and choose $r>0$ for which we have that $B_{d}(y, r) \subseteq K_{m}$. Proceed as previously but instead of taking $\varepsilon=\frac{1}{2(n+1)}-d\left(y, y_{s}\right)$ take $\varepsilon=\min \left\{\frac{1}{2(n+1)}-d\left(y, y_{s}\right), r\right\}>0$. Now notice that for any $y^{\prime} \in Y$ which satisfies $d\left(y, y^{\prime}\right)<\varepsilon$ we have that $y^{\prime} \in K_{m}$. So the resulting $y^{\prime} \in F\left(x^{\prime}\right)$ which witnesses that $d\left(y_{s}, F\left(x^{\prime}\right)\right) \leq \frac{1}{2(n+1)}$ is also a member of $K_{m}$. It follows that $d\left(y_{s}, F\left(x^{\prime}\right) \cap K_{m}\right) \leq \frac{1}{2(n+1)}$ as well. For the inverse direction replace $F(x)$ and $F\left(x^{\prime}\right)$ with the compact sets $F(x) \cap K_{m}$ and $F\left(x^{\prime}\right) \cap K_{m}$ respectively. Notice that when $d\left(y_{s}, F\left(x^{\prime}\right) \cap K_{m}\right)<1$ then -by our convention $d(y, \emptyset)=1$ - we have that $F\left(x^{\prime}\right) \cap K_{m} \neq \emptyset$. Having proved $(\dagger)$ one defines the set $R_{m, n, s}$ $(m, n, s \in \omega)$ as follows:

$$
x \in R_{m, n, s} \Longleftrightarrow \inf \left\{\sup \left\{d\left(y_{s}, F\left(x^{\prime}\right) \cap K_{m}\right) \mid x^{\prime} \in B_{p}(x, \delta)\right\} \quad \mid \delta>0\right\}<\frac{1}{n+1}
$$

and as before it is easy to see that the set $R_{m, n, s}$ is open. From $(\dagger)$ we have that $P=$ $\bigcup_{m} \bigcap_{n} R_{m, n, s}$ and so the set $P$ is $\Sigma_{3}^{0}$.

Readers who find that the convention $d(y, \emptyset)=1$ in the previous proof is rather artificial may notice that the condition

$$
\inf \left\{\sup \left\{d\left(y_{s}, F\left(x^{\prime}\right) \cap K_{m}\right) \mid x^{\prime} \in B_{p}(x, \delta)\right\} \mid \delta>0\right\}<\frac{1}{n+1}
$$


simply means that

$$
(\exists \delta>0)\left(\exists q \in \mathbb{Q}^{+}\right)\left(\forall x^{\prime} \in B_{p}(x, \delta)\right)\left[F\left(x^{\prime}\right) \cap K_{m} \neq \emptyset \& d\left(y_{s}, F\left(x^{\prime}\right) \cap K_{m}\right)<q<\frac{1}{n+1}\right] .
$$

Theorem 2.1 has an interesting corollary which answers to Question 63a) posed by M. Ziegler in [6].

Corollary 2.2. Suppose that $X$ is a metric space and that $F: X \Rightarrow \mathbb{R}^{m}$ is a multi-valued function such that the set $F(x)$ is closed for all $x \in X$.

(a) The set of the points of continuity of $F$ is $\Sigma_{3}^{0}$.

(b) If moreover the set $F(x)$ is bounded for all $x \in X$ then the set of points of continuity of $F$ is $\Pi_{2}^{0}$.

We now show that the results of Theorem 2.1 are optimum. It is well known that there are functions $f:[0,1] \rightarrow \mathbb{R}$ for which the set of points of continuity is not $F_{\sigma}$. Therefore the $\Pi_{2}^{0}$-answer is the best one can get. Thus we only need to deal with the $\Sigma_{3}^{0}$-answer. The following lemmas, although being straightforward from the definitions, will prove an elegant tool for the constructions that will follow.

Lemma 2.3. Suppose that $\left(X_{0}, p_{0}\right),\left(X_{1}, p_{1}\right),(Y, d)$ are metric spaces and that there exists a function $f: X_{0} \rightarrow X_{1}$ such that $f\left[X_{0}\right]$ is closed and $f: X_{0} \rightarrow f\left[X_{0}\right]$ is a topological isomorphism. Assume that we are given a multi-valued function $F: X_{0} \Rightarrow Y$. Define the multi-valued function $\tilde{F}: X_{1} \Rightarrow Y$ as follows:

$$
\tilde{F}\left(x_{1}\right)=F\left(x_{0}\right) \quad \text { if } x_{1}=f\left(x_{0}\right) \text { for some } x_{0} \in X_{0} \text { and } \quad \tilde{F}\left(x_{1}\right)=Y \quad \text { otherwise. }
$$

Then

(1) $\tilde{F}$ is continuous at $x_{1}$ if and only if either $x_{1} \notin f\left[X_{0}\right]$ or $x_{1}=f\left(x_{0}\right)$ and $F$ is continuous at $x_{0}$. Hence if we denote by $P_{0}$ and $P_{1}$ the set of points of continuity of $F$ and $\tilde{F}$ respectively we have that

$$
P_{1}=f\left[P_{0}\right] \cup\left(X_{1} \backslash f\left[X_{0}\right]\right) .
$$

(2) If $\Gamma$ is any of the classes $\Sigma_{n}^{0}, \Pi_{n}^{0}$, with $n \geq 2$ or $\Delta_{1}^{1}$ then

$$
P_{1} \in \Gamma \Longleftrightarrow P_{0} \in \Gamma \text {. }
$$

In particular if the set of points of continuity of $F$ is not $\Pi_{3}^{0}$ (Borel) then the set of points of continuity of $\tilde{F}$ is not $\Pi_{3}^{0}$ (Borel respectively).

Moreover the sets $F$ and $\tilde{F}$ as subsets of $X_{0} \times Y$ and $X_{1} \times Y$ respectively satisfy

$$
F \in \Gamma \Longleftrightarrow \tilde{F} \in \Gamma \text {. }
$$

(3) If $F\left(x_{0}\right)$ is a closed subset of $Y$ for all $x_{0} \in X_{0}$ then $\tilde{F}\left(x_{1}\right)$ is also a closed subset of $Y$ for all $x_{1} \in X_{1}$.

Proof. Clearly we only need to prove the first assertion. Suppose that $x_{1} \in X_{1} \backslash f\left[X_{0}\right]$. Since the set $f\left[X_{0}\right]$ is closed then there is some $\delta>0$ such that $B_{p_{1}}\left(x_{1}, \delta\right) \cap f\left[X_{0}\right]=\emptyset$. So for all $x_{1}^{\prime} \in B_{p_{1}}\left(x_{1}, \delta\right)$ we have that $\tilde{F}\left(x_{1}^{\prime}\right)=\tilde{F}\left(x_{1}\right)=Y$ and hence $\tilde{F}$ is continuous at $x_{1}$.

Assume that $x_{1}=f\left(x_{0}\right)$ where $x_{0}$ is a point of continuity of $F$. Let $y \in Y$ which witnesses the continuity of $F$ at $x_{0}$. We will show that this $y$ witnesses the continuity of $\tilde{F}$ at $x_{1}$. First notice that $F\left(x_{0}\right)=\tilde{F}\left(x_{1}\right)$ and so $y \in \tilde{F}\left(x_{1}\right)$. Assume now that we are given $\varepsilon>0$. Choose $\delta_{0}>0$ such that for all $x_{0}^{\prime} \in B_{p_{0}}\left(x_{0}, \delta_{0}\right)$ there is some $y^{\prime} \in F\left(x_{0}^{\prime}\right)$ such that 
$d\left(y, y^{\prime}\right)<\varepsilon$. Since the function $f^{-1}: f\left[X_{0}\right] \rightarrow X_{0}$ is continuous for this $\delta_{0}>0$ there is some $\delta_{1}>0$ such that for all $x_{1}^{\prime} \in B_{p_{1}}\left(x_{1}, \delta\right) \cap f\left[X_{0}\right]$ we have that $f^{-1}\left(x_{1}\right) \in B_{p_{0}}\left(x_{0}, \delta_{0}\right)$.

Let $x_{1}^{\prime} \in B_{p_{1}}\left(x_{1}, \delta_{1}\right)$. We claim that there is some $y^{\prime} \in \tilde{F}\left(x_{1}^{\prime}\right)$ such that $d\left(y, y^{\prime}\right)<\varepsilon$. We take cases: if $x_{1}^{\prime} \notin f\left[X_{0}\right]$ then $\tilde{F}\left(x_{1}^{\prime}\right)=Y$ and so we may take $y^{\prime}=y$; if $x_{1}^{\prime}=f\left(x_{0}^{\prime}\right)$ for some $x_{0}^{\prime} \in X_{0}$, then $x_{0}^{\prime}$ belongs to $B_{p_{0}}\left(x_{0}, \delta_{0}\right)$ and so there is some $y^{\prime} \in F\left(x_{0}^{\prime}\right)=\tilde{F}\left(x_{1}^{\prime}\right)$ such that $d\left(y, y^{\prime}\right)<\varepsilon$.

Now assume that $x_{1}=f\left(x_{0}\right)$ and $x_{0}$ is not a point of continuity of $F$. Let $y \in \tilde{F}\left(x_{1}\right)=$ $F\left(x_{0}\right)$. Since $F$ is not continuous at $x_{0}$ there is some $\varepsilon^{*}>0$ such that for all $\delta_{0}>0$ there is some $x_{0}^{\prime} \in B_{p_{0}}\left(x_{0}, \delta_{0}\right)$ such that for all $y^{\prime} \in F\left(x_{0}^{\prime}\right)$ we have that $d\left(y, y^{\prime}\right) \geq \varepsilon^{*}$. We claim that this $\varepsilon^{*}$ witnesses that $\tilde{F}$ is not continuous at $x_{1}$.

Let $\delta_{1}$; since $f$ is continuous there is some $\delta_{0}>0$ such that for all $x_{0}^{\prime} \in B_{p_{0}}\left(x_{0}, \delta_{0}\right)$ we have that $f\left(x_{0}^{\prime}\right) \in B_{p_{1}}\left(x_{1}, \delta_{1}\right)$. Choose $x_{0}^{\prime} \in B_{p_{0}}\left(x_{0}, \delta_{0}\right)$ such that for all $y^{\prime} \in F\left(x_{0}^{\prime}\right)$ we have that $d\left(y, y^{\prime}\right) \geq \varepsilon^{*}$. Let $x_{1}^{\prime}=f\left(x_{0}^{\prime}\right)$; then $x_{1}^{\prime} \in B_{p_{1}}\left(x_{1}, \delta_{1}\right)$ and also for all $y^{\prime} \in \tilde{F}\left(x_{1}^{\prime}\right)=F\left(x_{0}^{\prime}\right)$ we have that $d\left(y, y^{\prime}\right) \geq \varepsilon^{*}$.

Lemma 2.3 has a cute corollary which might be regarded as the multi-valued analogue of the Tietze Extension Theorem.

Corollary 2.4. Every continuous multi-valued function which is defined on a closed subset of a metric space can be extended continuously on the whole space.

Lemma 2.5. Let $X, Y, Z$ be metric spaces, $F: X \Rightarrow Y$ be a multi-valued function and $\pi: Y \rightarrow Z$ be a topological isomorphism between $Y$ and $\pi[Y]$. Define the composition $\pi \circ F: X \Rightarrow Z:$

The following hold.

$$
(\pi \circ F)(x)=\pi[F(x)], \quad x \in X
$$

(1) A point $x \in X$ is a point of continuity of $F$ if and only if $x$ is a point of continuity of $\pi \circ F$

(2) If the set $\pi[Y]$ is closed and the set $F(x)$ is closed for some $x \in X$ then the $\operatorname{set}(\pi \circ F)(x)$ is also closed.

(3) If $F$ is a Borel subset of $X \times Y$ then $\pi \circ F$ is a Borel subset of $X \times Z$.

Proof. The first assertion is proved as Lemma 2.3 using the following remarks. For all $z=\pi(y)$ and for all $\varepsilon^{*}>0$ there is some $\varepsilon>0$ such that for all $y^{\prime} \in B_{Y}(y, \varepsilon)$ we have that $\pi\left(y^{\prime}\right) \in B_{Z}\left(z, \varepsilon^{*}\right)$; and for all $y \in Y$ and for all $\varepsilon>0$ there is some $\varepsilon^{*}>0$ such that for all $z^{\prime} \in B_{Z}\left(\pi(y), \varepsilon^{*}\right) \cap \pi[Y]$ we have that $\pi^{-1}(z) \in B_{Y}(y, \varepsilon)$.

For the second assertion we know that if $F(x)$ is closed then since $\pi$ is a topological isomorphism the set $\pi[F(x)]$ is closed in $\pi[Y]$. So if moreover $\pi[Y]$ is closed in $Z$ it follows that $\pi[F(x)]$ is closed in $Z$.

For the third assertion notice that

$$
(x, z) \in \pi \circ F \Longleftrightarrow z \in \pi[Y] \&\left(x, \pi^{-1}(z)\right) \in F .
$$

and so $\pi \circ F=(X \times \pi[Y]) \cap h^{-1}[F]$, where $h(x, y)=(x, \pi(y))$. From 2E.9 of [5] we have that the set $\pi[Y]$ is Borel and hence $\pi \circ F$ is Borel.

Theorem 2.6. There is a multi-valued function $F:[0,1] \rightarrow \mathbb{R}$ such that the set $F(x)$ is closed for all $x$, the set $F$ is a $\Pi_{2}^{0}$ subset of $[0,1] \times \mathbb{R}$ and the set of points of continuity of $F$ is not $\Pi_{3}^{0}$. Therefore the $\Sigma_{3}^{0}$-answer is the best possible for a multi-valued function $F$ from $[0,1]$ to $\mathbb{R}$ even if $F$ is below the $\Sigma_{3}^{0}$-level. 
Using the fact that the set of points of continuity of a function $f:(X, p) \rightarrow(Y, d)$ is a $G_{\delta}$ set, we conclude to the following.

Corollary 2.7. The notion of continuity which is given in Definition 1.1 for a multi-valued function $F:(X, p) \Rightarrow(Y, d)$ cannot be reduced to the usual notion of continuity if we view $F$ as a single-valued function from $X$ to the powerset $\mathcal{P}(Y)$ of $Y$. To be more specific it is not true in general that if we are given a multi-valued function $F:(X, p) \Rightarrow(Y, d)$ there is a metric $d_{F}$ on the set $F[X]=\{F(x) \mid x \in X\}$ such that for all $x \in X$, the function $F:(X, p) \rightarrow\left(F[X], d_{F}\right)$ is continuous at $x$ in the usual sense exactly when $F$ is continuous at $x$ in the sense of Definition 1.1.

Now we proceed with the proof of Theorem 2.6.

Proof. Since $2^{\omega \times \omega}$ is isomorphic with the space $\mathcal{C}$, from Lemma 2.3 it is enough to define a multi-valued function $F: 2^{\omega \times \omega} \Rightarrow \mathbb{R}$ such that the set $F(\gamma)$ is closed for all $\gamma \in 2^{\omega \times \omega}$, the set $F$ is $\Pi_{2}^{0}$ and also the set of points of continuity of $F$ is not $\Pi_{3}^{0}$.

A typical example of a $\Sigma_{3}^{0}$ set which is not $\Pi_{3}^{0}$ is the following:

$$
R=\left\{\gamma \in 2^{\omega \times \omega} \mid(\exists m)(\forall n)(\exists s \geq n)[\gamma(m, s)=1]\right\},
$$

(see 4] 23.1). For all $m \in \omega$ define $R_{m}$ as the set of all $\gamma \in 2^{\omega \times \omega}$ such that for all $n$ there is some $s \geq n$ such that $\gamma(m, s)=1$, so that $R=\bigcup_{m} R_{m}$.

If for some $m$ and $\gamma$ we have that $\gamma$ is not a member of $R_{m}$, then there is some $n$ such that for all $s \geq n$ we have that $\gamma(m, s)=0$. For $\gamma \notin R_{m}$ put

$$
n(\gamma, m)=\text { the least } n \in \omega[\text { for all } s \geq n \text { we have that } \gamma(m, s)=0]+1 \text {. }
$$

Define $F: 2^{\omega \times \omega} \Rightarrow \mathbb{R}$ as follows

$$
F(\gamma)=\left\{m+\frac{1}{n(\gamma, m)+1} \mid \gamma \notin R_{m}\right\} \cup\left\{m \mid \gamma \in R_{m}\right\}
$$

Notice that $n(\gamma, m)+1 \geq 2$ for all $\gamma \notin R_{m}$. Clearly the set $F(\gamma)$ is closed for all $\gamma \in 2^{\omega \times \omega}$. First we prove that the set $F$ is a $\Pi_{2}^{0}$ subset of $2^{\omega \times \omega} \times \mathbb{R}$. Indeed

$$
\begin{aligned}
(\gamma, y) \in F \Longleftrightarrow & (\exists m \leq y)\{\text { either }[y=m \&(\forall n)(\exists s \geq n)[\gamma(m, s)=1]] \\
& \text { or }\left[y \neq m \& \frac{1}{y-m}-2 \in \omega \&\left(\forall s \geq \frac{1}{y-m}-2\right)[\gamma(m, s)=0]\right. \\
& \left.\left.\&\left(\forall i<\frac{1}{y-m}-2\right)(\exists s>i)[\gamma(m, s)=1]\right]\right\} .
\end{aligned}
$$

We now claim that

$$
\gamma \in R \Longleftrightarrow F \text { is continuous at } \gamma \text {. }
$$

Let $\gamma \in R$. Fix some $m \in \omega$ such that for all $n$ there is some $s \geq n$ such that $\gamma(m, s)=1$ i.e., $\gamma \in R_{m}$. We claim that the natural $m$ which belongs to $F(\gamma)$ witnesses the continuity of $F$ at $\gamma$. Let $\varepsilon>0$ and $n \in \omega$ such that $\frac{1}{n+1}<\varepsilon$. Since $\gamma \in R_{m}$ for this $n$ we can choose some $s_{n} \geq n$ such that $\gamma\left(m, s_{n}\right)=1$. Define

$$
W=\left\{\beta \in 2^{\omega \times \omega} \quad \mid \beta\left(m, s_{n}\right)=\gamma\left(m, s_{n}\right)=1\right\} .
$$

Clearly $W$ is a basic neighborhood of $\gamma$. We will prove that for all $\beta \in W$ there is some $y^{\prime} \in F(\beta)$ such that $\left|m-y^{\prime}\right|<\varepsilon$. Indeed if $\beta \in W$ and $\beta \in R_{m}$ then $m \in F(\beta)$ so one can take $y^{\prime}=m$; if $\beta \notin R_{m}$ since $\beta\left(m, s_{n}\right)=\gamma\left(m, s_{n}\right)=1$ we have that $n(\beta, m)>s_{n}$. 
Hence for $y^{\prime}=m+\frac{1}{n(\beta, m)+1}$ we have that $y^{\prime} \in F(\beta)$ (because $\beta \notin R_{m}$ ) and also that $\left|m-y^{\prime}\right|=\frac{1}{n(\beta, m)+1}<\frac{1}{s_{n}+1} \leq \frac{1}{n+1}<\varepsilon$. Therefore $F$ is continuous at $\gamma$.

Assume now that $\gamma \notin R$; clearly $\gamma \notin R_{m}$ for all $m \in \omega$. From the definition it follows that $F(\gamma)=\left\{m+\frac{1}{n(\gamma, m)+1} \mid m \in \omega\right\}$. Let any $y \in F(\gamma)$; then $y=m+\frac{1}{n(\gamma, m)+1}$ for some $m \in \omega$. Since $n(\gamma, m)+1 \geq 2$ it follows that $y=m+\frac{1}{n(\gamma, m)+1} \leq m+\frac{1}{2}$. Hence for $\varepsilon=\frac{1}{2} \cdot \frac{1}{n(\gamma, m)+1}>0$ we have that $\left|y-y^{\prime}\right| \geq \varepsilon$ for all $y^{\prime} \in \omega$. We will show that for all basic neighborhoods $W$ of $\gamma$ there is some $\beta \in W$ such that for all $y^{\prime} \in F(\beta)$ we have that $\left|y-y^{\prime}\right| \geq \varepsilon$. Let $V$ be any basic neighborhood of $\gamma$. Then there are some naturals $m_{0}, \ldots, m_{k}, s_{0}, \ldots, s_{k}$ such that

$$
V=\left\{\beta \in 2^{\omega \times \omega} \quad \mid \forall i \leq k \beta\left(m_{i}, s_{i}\right)=\gamma\left(m_{i}, s_{i}\right)\right\}
$$

Define $\beta \in 2^{\omega \times \omega}$ as follows: $\beta\left(m_{i}, s_{i}\right)=\gamma\left(m_{i}, s_{i}\right)$ for all $i \leq k$; and $\beta(m, s)=1$ in any other case. It is clear that $\beta \in V$ and also that $\beta \in R_{m}$ for all $m \in \omega$. Hence $F(\beta)=\omega$ and therefore for any $y^{\prime} \in F(\beta)$ we have that $\left|y-y^{\prime}\right| \geq \varepsilon$.

The case of analytic sets. One can ask what is the best that we can say about the set of points of continuity of $F$ without any additional topological assumptions for $Y$ or for $F(x)$. The following proposition gives an upper bound for the complexity of this set. (Notice though that we restrict ourselves to complete and separable metric spaces.)

Proposition 2.8. Let $(X, p)$ and $(Y, d)$ be complete and separable metric spaces and let $F: X \Rightarrow Y$ be a multi-valued function such that the set $F \subseteq X \times Y$ is analytic. Then the set of points of continuity of $F$ is analytic as well.

Proof. Following the proof of Theorem 2.1 for all $n \in \omega$ we define the set $A_{n} \subseteq X \times Y$ as follows

$$
(x, y) \in A_{n} \Longleftrightarrow \inf \left\{\sup \left\{d\left(y, F\left(x^{\prime}\right)\right) \mid x^{\prime} \in B_{p}(x, \delta)\right\} \mid \delta>0\right\}<\frac{1}{n+1} .
$$

Also we denote by $P$ the set of points of continuity of $F$. It is clear that

$$
\begin{aligned}
x \in P & \Longleftrightarrow(\exists y \in F(x))(\forall n)\left[(x, y) \in A_{n}\right] \\
& \Longleftrightarrow(\exists y)\left[(x, y) \in F \&(\forall n)\left[(x, y) \in A_{n}\right]\right]
\end{aligned}
$$

We claim that every set $A_{n}$ is open. This is again straightforward from the definitions and the fact that

$$
\left|d\left(y_{1}, B\right)-d\left(y_{2}, B\right)\right| \leq d\left(y_{1}, y_{2}\right)
$$

for all non-empty sets $B \subseteq Y$. Fix $n \in \omega$ and let $(x, y) \in A_{n}$. Choose $\varepsilon_{0}, \delta_{0}>0$ such that

$$
\sup \left\{d\left(y, F\left(x^{\prime}\right)\right) \mid x^{\prime} \in B_{p}\left(x, \delta_{0}\right)\right\}+\varepsilon_{0}<\frac{1}{n+1}
$$

We will show that for all $\left(x_{1}, y_{1}\right) \in X \times Y$ with $x_{1} \in B_{p}\left(x, \delta_{0}\right)$ and $y_{1} \in B_{d}\left(y, \varepsilon_{0}\right)$ we have that $\left(x_{1}, y_{1}\right) \in A_{n}$. Let $x_{1} \in B_{p}\left(x, \delta_{0}\right)$ and $y_{1} \in B_{d}\left(y, \varepsilon_{0}\right)$; choose $\delta_{1}>0$ such that 
$B_{p}\left(x_{1}, \delta_{1}\right) \subseteq B_{p}\left(x, \delta_{0}\right)$. We compute

$$
\begin{aligned}
& \inf \left\{\sup \left\{d\left(y_{1}, F\left(x^{\prime}\right)\right) \mid x^{\prime} \in B_{p}\left(x_{1}, \delta\right)\right\} / \delta>0\right\} \\
\leq & \sup \left\{d\left(y_{1}, F\left(x^{\prime}\right)\right) \mid x^{\prime} \in B_{p}\left(x_{1}, \delta_{1}\right)\right\} \\
\leq & \sup \left\{d\left(y_{1}, F\left(x^{\prime}\right)\right) \mid x^{\prime} \in B_{p}\left(x, \delta_{0}\right)\right\} \\
\leq & \sup \left\{d\left(y, F\left(x^{\prime}\right)\right) \mid x^{\prime} \in B_{p}\left(x, \delta_{0}\right)\right\}+d\left(y_{1}, y\right) \\
< & \sup \left\{d\left(y, F\left(x^{\prime}\right)\right) \quad \mid x^{\prime} \in B_{p}\left(x, \delta_{0}\right)\right\}+\varepsilon_{0}<\frac{1}{n+1}
\end{aligned}
$$

i.e., $(x, y) \in A_{n}$ and the set $A_{n}$ is open. Now define $A=\cap A_{n}$; from (1) above we have that

$$
x \in P \Longleftrightarrow(\exists y)[(x, y) \in F \cap A] \text {, }
$$

so that the set $P$ is the projection along $Y$ of the analytic set $F \cap A$. It follows that $P$ is analytic.

Now we show that if we remove just one of our assumptions about $F(x)$ or about $Y$ in Theorem 2.1, then it is possible that the set of points of continuity of $F$ is not even a Borel set. Therefore Proposition 2.8 is the best that one can say in the general case.

\section{Theorem 2.9.}

(a) There is a multi-valued function $F: \mathcal{C} \Rightarrow \mathcal{N}$ such that the set $F(x)$ is closed for all $x \in \mathcal{C}$ and the set of points of continuity of $F$ is analytic and not Borel. Moreover the set $F$ is a Borel subset of $\mathcal{C} \times \mathcal{N}$.

(b) There is a multi-valued function $F:[0,1] \Rightarrow[0,1]$ for which the set of the points of continuity of $F$ is analytic and not Borel. Moreover the set $F$ is a Borel subset of $[0,1] \times[0,1]$.

Before proving this theorem it is perhaps useful to make the following remarks. If we replace in (a) of Theorem 2.1 the condition about $F(x)$ being compact for all $x$ with " $F(x)$ is closed for all $x$ ", then from (a) of Theorem 2.9 we can see that the result fails in the worst possible way. Also -in connection with (b) of Theorem 2.1 we can see that if we drop the hypothesis about $Y$ being exhaustible by compact sets but keep the second condition " $F(x)$ is closed for all $x$ ", then again the result fails in the worst possible way.

If we replace in (a) of Theorem 2.1 the hypothesis " $F(x)$ is compact for all $x$ ", with " $Y$ is compact" then still the result fails in the worst possible way.

In conclusion if we want to obtain that the set of points of continuity of a multi-valued function $F$ is Borel, then we cannot drop the condition " $F(x)$ is closed for all $x$ ". But yet this condition alone is not sufficient in order to derive this result as long as $Y$ is neither compact nor exhaustible by compact sets.

Proof. We begin with (a). Let $\operatorname{Tr}$ be the set of all (non-empty) trees on $\omega$, (see the Introduction). As we mentioned before the set $\operatorname{Tr}$ can be regarded as a compact subspace of the Cantor space $\mathcal{C}$. From Lemma 2.3 it is enough to construct a multi-valued function $F: \operatorname{Tr} \Rightarrow \mathcal{N}$ such that the set of points of continuity of $F$ is not Borel, the set $F(T)$ is closed for all $T \in T r$ and the set $F$ is a Borel subset of $\operatorname{Tr} \times \mathcal{N}$.

Denote by $I F$ the set of all ill founded trees i.e, the set of all $T \in T r$ for which the body $[T]$ is not empty. It is well known (see [4 27.1) that the set $I F$ is an analytic subset 
of $\operatorname{Tr}$ which is not Borel 2 For $T \in T r$ we define the tree

$$
T^{+1}=\{(u(0)+1, \ldots, u(n-1)+1) \mid u \in T, \operatorname{lh}(u)=n\} .
$$

Also we define the set $\operatorname{trm}(T)$ as the set of all terminal nodes of $T$ i.e., the set of all those $u$ 's in $T$ for which there is no $w \in T$ such that $u \sqsubseteq w$ and $u \neq w$. Define the multi-valued function $F: \operatorname{Tr} \Rightarrow \mathcal{N}$ as follows

$$
F(T)=\left[T^{+1}\right] \cup\left\{u^{\wedge}(0,0,0, \ldots) \mid u \in \operatorname{trm}\left(T^{+1}\right)\right\}
$$

for all $T \in T r$. Notice that if some $T \in T r$ has no terminal nodes then $T$ must have at least one infinite branch i.e., if $\operatorname{trm}(T)=\emptyset$ then $[T] \neq \emptyset$. So $F(T) \neq \emptyset$ for all $T \in \operatorname{Tr}$ i.e., the function $F$ is total.

First we prove that $F$ is a Borel subset of $\mathcal{C} \times \mathcal{N}$. Indeed compute

$$
\begin{aligned}
(T, \beta) \in F \Longleftrightarrow & \text { either }[(\forall n) \beta(n) \geq 1 \&(\forall n)(\beta(0)-1, \ldots, \beta(n-1)-1) \in T] \\
& \text { or }[(\exists n)(\forall m \geq n)(\forall i<n) \\
& \{\beta(m)=0 \& \beta(i) \geq 1 \&(\beta(0)-1, \ldots, \beta(n-1)-1) \in T \\
& \quad \&(\forall u)[(u \in T \& l h(u)>n) \rightarrow(\exists j<n)[\beta(j)-1 \neq u(j)]]\}] .
\end{aligned}
$$

(The last line is to say that $(\beta(0)-1, \ldots, \beta(n-1)-1)$ is terminal in $T$ ). It follows that $F$ is a $\Sigma_{2}^{0}$ set. From this and Proposition 2.8 we get that the set of points of continuity of $F$ is analytic.

Now we prove that for all $T \in T r$ the set $F(T)$ is a closed subset of the Baire space $\mathcal{N}$. Let $\left(\alpha_{i}\right)_{i \in \omega}$ be a sequence in $F(T)$ which converges to some $\alpha$. We will prove that $\alpha \in F(T)$. If $\alpha_{i} \in\left[T^{+1}\right]$ for infinitely many $i$ 's then since $\left[T^{+1}\right]$ is a closed set we have that $\alpha \in\left[T^{+1}\right]$ as well. So assume that

$$
\alpha_{i}=u_{i}{ }^{\wedge}(0,0, \ldots) \text { for some } u_{i} \in \operatorname{trm}\left(T^{+1}\right) \text { for all } i \in \omega \text {. }
$$

We distinguish cases. In the first case we have that $\sup \left\{\operatorname{lh}\left(u_{i}\right) \mid i \in \omega\right\}<\infty$. Then there is a subsequence $\left(u_{k_{i}}\right)_{i \in \omega}$ and some $n_{0} \in \omega$ such that $l h\left(u_{k_{i}}\right)=n_{0}$ for all $i \in \omega$. Hence for all $i \in \omega$

$$
\alpha_{k_{i}}(t)=u_{k_{i}}(t) \text { for all } t<n_{0} \text { and } \alpha_{k_{i}}(t)=0 \text { for all } t \geq n_{0} \quad(2)
$$

Since the sequence $\left(\alpha_{k_{i}}(t)\right)_{i \in \omega}$ is convergent for all $t \in \omega$ it follows from (2) that the sequence $\left(u_{k_{i}}(t)\right)_{i \in \omega}$ is also convergent for all $t<n_{0}$. Define $u(t)=\lim _{i \in \omega} u_{k_{i}}(t)$ for all $t<n_{0}$. Clearly $u$ is a finite sequence of natural numbers with length equal to $n_{0} 3$ and there is some $i_{0}$ such that for all $i \geq i_{0}$ and for all $t<n_{0}$ we have that $u_{k_{i}}(t)=u(t)$ i.e.,

$$
u_{k_{i}}=u \text { for all } i \geq i_{0} \text {. }
$$

Since $u_{k_{i_{0}}} \in \operatorname{trm}\left(T^{+1}\right)$ we have that $u \in \operatorname{trm}\left(T^{+1}\right)$ as well. We will show that $\alpha=$ $u^{\wedge}(0,0, \ldots)$. Let $n \in \omega$; since $\alpha_{k_{i}} \rightarrow \alpha$ then there is some $i_{1} \geq i_{0}$ such that $\alpha_{k_{i_{1}}}(n)=\alpha(n)$. If $n<n_{0}=l h(u)$ then -using (2) and (3)- we have that

$$
\alpha(n)=\alpha_{k_{i_{1}}}(n)=u_{k_{i_{1}}}(n)=u(n)
$$

and if $n \geq n_{0}$ then again from (2)

$$
\alpha(n)=\alpha_{k_{i_{1}}}(n)=0
$$

\footnotetext{
${ }^{2} \mathrm{~A}$ classical way for proving that a given set $A \subseteq \mathcal{X}$ is not Borel is finding a Borel function $\pi: \operatorname{Tr} \rightarrow \mathcal{X}$ such that $I F=\pi^{-1}[A]$. If $A$ was a Borel set then $I F$ would be Borel, a contradiction.

${ }^{3}$ If $n_{0}=0$ then $u_{k_{i}}$ is the empty sequence for all $i \geq i_{0}$. In this case we let $u$ be the empty sequence as well.
} 
Hence $\alpha=u^{\wedge}(0,0, \ldots)$ and since $u \in \operatorname{trm}\left(T^{+1}\right)$ we also have that $\alpha \in F(T)$. In the second case we have that $\sup \left\{l h\left(u_{i}\right) \mid i \in \omega\right\}=\infty$. Choose a subsequence $\left(u_{k_{i}}\right)_{i \in \omega}$ such that $l h\left(u_{k_{i}}\right) \geq i$ for all $i \in \omega$. Now we claim that $\alpha \in\left[T^{+1}\right]$. Let $n \in \omega$; since $\alpha_{k_{i}} \rightarrow \alpha$ there is some $i_{0} \in \omega$ such that for all $i \geq i_{0}$ and for all $t<n$ we have that $\alpha_{k_{i}}(t)=\alpha(t)$. For $i>n>t$ since $l h\left(u_{k_{i}}\right) \geq k_{i} \geq i>n>t$ from (1) we have that $\alpha_{k_{i}}(t)=u_{k_{i}}(t)$. So if we fix some $i_{1}>\max \left\{i_{0}, n\right\}$ then for all $t<n$ we have that $\alpha(t)=\alpha_{k_{i_{1}}}(t)=u_{k_{i_{1}}}(t)$ i.e.,

$$
\left.(\alpha(0), \ldots, \alpha(n-1))=\left(u_{k_{i_{1}}}(0), \ldots, u_{k_{i_{1}}}(n-1)\right) \in T^{+1} \quad \text { (because } u_{k_{i_{1}}} \in T^{+1}\right) .
$$

So we have proved that $(\alpha(0), \ldots, \alpha(n-1)) \in T^{+1}$ for all $n \in \omega$ i.e., $\alpha \in\left[T^{+1}\right]$.

Thus the set $F(T)$ is closed for all $T \in T r$. Now we prove that for $T \in T r$

$F$ is continuous at $T \Longleftrightarrow T \in I F$.

Since $I F$ is not Borel if we prove the above equivalence we are done. Assume that $T$ is not in $I F$ and so $[T]=\left[T^{+1}\right]=\emptyset$. Let any $\alpha \in F(T)$; then there is some $u$ which is terminal in $T$ such that

$$
\alpha=\left(u(0)+1, \ldots, u\left(n_{0}-1\right)+1\right)^{\wedge}(0,0,0, \ldots)
$$

where $n_{0}=l h(u)$. Define

$$
V=\left\{\beta \in \mathcal{N} \mid \forall i \leq n_{0} \beta(i)=\alpha(i)\right\}
$$

Clearly $V$ is a basic neighborhood of $\alpha$. Let $W$ be any basic neighborhood of $T$ and let $u_{0}, \ldots, u_{n-1} \in T, w_{0}, \ldots w_{m-1} \in \omega^{\star} \backslash T$ be such that

$$
W=\left\{T^{\prime} \in \operatorname{Tr} \mid(\forall i<n)\left[u_{i} \in T^{\prime}\right] \&(\forall j<m)\left[w_{j} \notin T^{\prime}\right]\right\} .
$$

We will prove that there is some $T^{\prime} \in W$ such that for all $\alpha^{\prime} \in F\left(T^{\prime}\right)$ we have that $\alpha^{\prime} \notin V$. Since $T \in W$ and $u \in T$ we may assume (by moving to a subset of $W$ if necessary) that $u_{0}=u$. Also we may assume that for all $1 \leq i<n$ the finite sequence $u_{i}$ is not an initial segment of $u_{0}$; (for otherwise we just remove it from the list - clearly the set $W$ is not affected).

Notice that since $T \in W$ it is not possible to have $w_{j} \sqsubseteq u_{i}$ for any $i<n$ and $j<m$.

Fix some $t_{0} \in \omega$ such that $u_{0}{ }^{\wedge}\left(t_{0}\right) \neq w_{j}$ for all $j<m$. It follows that for all $j<m$ the finite sequence $w_{j}$ is not an initial segment of $u_{0} \wedge\left(t_{0}\right)$; for otherwise we would have that $w_{j} \sqsubseteq u_{0}$ for some $j<m$, a contradiction. Let $T^{\prime}$ be the tree which is generated by the set $\left\{u_{0} \wedge\left(t_{0}\right), u_{1}, \ldots, u_{n-1}\right\}$ (look at the Introduction). Clearly the tree $T^{\prime}$ is finite and $u_{i} \in T^{\prime}$ for all $i<n$. If it where $w_{j} \in T^{\prime}$ for some $j<m$ then since $w_{j}$ is not an initial segment of $u_{0} \wedge\left(t_{0}\right)$ there would be some $1 \leq i<n$ such that $w_{j} \sqsubseteq u_{i}$, a contradiction. Hence $w_{j} \notin T^{\prime}$ for all $j<m$. It follows that $T^{\prime} \in W$.

Now let any $\alpha^{\prime} \in F\left(T^{\prime}\right)$. Since $T^{\prime}$ has empty body there is some $u^{\prime}$ which is terminal in $T^{\prime}$ such that

$$
\alpha^{\prime}=\left(u^{\prime}(0)+1, \ldots, u^{\prime}\left(n_{1}-1\right)+1\right)^{\wedge}(0,0,0, \ldots)
$$

where $n_{1}=l h\left(u^{\prime}\right)$. Since $u^{\prime}$ is terminal in $T^{\prime}$ it follows that $u^{\prime} \in\left\{u_{0} \wedge^{\wedge}\left(t_{0}\right), u_{1}, \ldots, u_{n-1}\right\}$. If $u^{\prime}=u_{0} \wedge\left(t_{0}\right)=u^{\wedge}\left(t_{0}\right)$ then $u^{\prime}\left(n_{1}-1\right)=t_{0}$ and also $\operatorname{lh}\left(u^{\prime}\right)=\operatorname{lh}(u)+1$ i.e., $n_{1}=n_{0}+1$. It follows that $\alpha^{\prime}\left(n_{0}\right)=\alpha^{\prime}\left(n_{1}-1\right)=u^{\prime}\left(n_{1}-1\right)+1=t_{0}+1>0$. But on the other hand $\alpha\left(n_{0}\right)=0$ and so $\alpha^{\prime} \notin V$. If $u^{\prime}=u_{i}$ for some $1 \leq i<n$ then since $u_{i}$ is not an initial segment of $u_{0}=u$ and $u_{0}$ is terminal in $T$ we have that the finite sequences $u$ and $u^{\prime}$ are incompatible. Hence there some $k<\min \left\{l h\left(u^{\prime}\right), \operatorname{lh}(u)\right\}$ such that $u(k) \neq u^{\prime}(k)$. Since $k<\min \left\{l h\left(u^{\prime}\right), \operatorname{lh}(u)\right\}$ we have that $\alpha^{\prime}(k)=u^{\prime}(k)+1 \neq u(k)+1=\alpha(k)$ i.e., there is some $k<n_{0}=\operatorname{lh}(u)$ such that $\alpha^{\prime}(k) \neq \alpha(k)$. Hence $\alpha^{\prime} \notin V$. 
Now assume that $T \in I F$. We will prove that $F$ is continuous at $T$. Let $\alpha$ be any member of $\left[T^{+1}\right]$ and let $V$ be a basic neighborhood of $\alpha$ i.e., for some fixed $n_{0}$ we have that

Define

$$
V=\left\{\beta \in \mathcal{N} \mid \forall i \leq n_{0} \beta(i)=\alpha(i)\right\}
$$

$$
W=\left\{T \in \operatorname{Tr} \mid\left(\alpha(0)-1, \ldots, \alpha\left(n_{0}\right)-1\right) \in T\right\} .
$$

Since $\alpha \in\left[T^{+1}\right]$ we have that $T \in W$. We will prove that for all $T^{\prime} \in W$ there is some $\alpha^{\prime} \in F\left(T^{\prime}\right)$ such that $\alpha^{\prime} \in V$. Indeed let $T^{\prime} \in W$, then $\left(\alpha(0)-1, \ldots, \alpha\left(n_{0}\right)-1\right) \in T^{\prime}$. There are two cases: either there is some $u \in T^{\prime}$ with $\left(\alpha(0)-1, \ldots, \alpha\left(n_{0}\right)-1\right) \sqsubseteq u$ and $u$ is terminal in $T^{\prime}$, or there is some $\beta \in\left[T^{\prime}\right]$ such that $\left(\alpha(0)-1, \ldots, \alpha\left(n_{0}\right)-1\right)=\left(\beta(0), \ldots, \beta\left(n_{0}\right)\right)$.

Assume the first case i.e., there is some $u \in T^{\prime}$ such that $\left(\alpha(0)-1, \ldots, \alpha\left(n_{0}\right)-1\right) \sqsubseteq u$ and $u$ is terminal in $T^{\prime}$. Clearly $l h(u) \geq n_{0}+1$. Take

$$
\alpha^{\prime}=\left(u(0)+1, \ldots, u\left(n_{1}-1\right)+1\right)^{\wedge}(0,0, \ldots)
$$

where $n_{1}=l h(u) \geq n_{0}+1$. Since $u$ is terminal in $T^{\prime}$ we have that $\alpha^{\prime} \in F\left(T^{\prime}\right)$. Also for all $i \leq n_{0}<n_{1}$ we have that $\alpha^{\prime}(i)=u(i)+1$; since $\left(\alpha(0)-1, \ldots, \alpha\left(n_{0}\right)-1\right) \sqsubseteq u$ it follows that $\alpha(i)-1=u(i)$ and so $\alpha(i)=u(i)+1=\alpha^{\prime}(i)$. Hence for all $i \leq n_{0}$ we have that $\alpha^{\prime}(i)=\alpha(i)$ i.e., $\alpha^{\prime} \in V$.

Assume the second case i.e., there is some $\beta \in\left[T^{\prime}\right]$ such that $\left(\alpha(0)-1, \ldots, \alpha\left(n_{0}\right)-1\right)=$ $\left(\beta(0), \ldots, \beta\left(n_{0}\right)\right)$. Take $\alpha^{\prime}(i)=\beta(i)+1$ for all $i \in \omega$. Since $\beta \in\left[T^{\prime}\right]$ we have that $\alpha^{\prime} \in\left(T^{\prime}\right)^{+1} \subseteq F\left(T^{\prime}\right)$. Also for all $i \leq n_{0}$ we have that $\alpha^{\prime}(i)=\beta(i)+1=\alpha(i)$. Therefore $\alpha^{\prime} \in V$ and we are done. This proves assertion $(a)$.

Assertion $(b)$ is an easy consequence of the previous. From $(a)$ and Lemma 2.3 it follows that there is a multi-valued function $F:[0,1] \Rightarrow \mathcal{N}$ such that the set of points of continuity of $F$ is analytic and not Borel. Moreover the set $F$ is a Borel subset of $[0,1] \times \mathcal{N}$. It is well known that there is a topological isomorphism between $\mathcal{N}$ and a subset of $[0,1]$; we give a description of its construction. By induction one constructs a family $\left(I_{u}\right)_{u \in \omega^{\star}}$ of closed intervals such that $I_{\langle\cdot\rangle}=[0,1] ; \operatorname{length}\left(I_{u}\right) \leq \frac{1}{2^{l h(u)}}$ for all $u ; I_{u \wedge}(n) \subseteq I_{u}$ for all $u, n$; and $I_{u} \cap I_{w}=\emptyset$ for all incompatible $u, w$. It is not hard to see that the function $\pi: \mathcal{N} \rightarrow[0,1]$ defined by $\pi(\alpha)=$ the unique $x \in[0,1]$ such that $x \in \bigcap_{n} I_{(\alpha(0), \ldots, \alpha(n))}$ is a topological isomorphism between $\mathcal{N}$ and a $G_{\delta}$ subset of $[0,1]$.

Since there is a topological isomorphism between $\mathcal{N}$ and a subset of $[0,1]$ from the first assertion of Lemma 2.5 there is a multi-valued function $G:[0,1] \Rightarrow[0,1]$ for which the set of points of continuity is analytic and not Borel. Moreover from the third assertion of Lemma 2.5 the set $G$ is a Borel subset of $[0,1] \times[0,1]$ [4

\section{Question 1.}

(1) In all theorems and examples we have seen about multi-valued functions, the set of points of continuity is either a $\Sigma_{3}^{0}$ set or not even a Borel set. It would be interesting to see if one can construct multi-valued functions, whose set of points of continuity lies somewhere between the $\Sigma_{3}^{0}$-level and the level of analytic sets. More specifically: does there exist for every $n>3$ a multi-valued function, whose set of points of continuity is a $\Sigma_{n}^{0}\left(\right.$ or $\left.\Pi_{n}^{0}\right)$ and not a $\Pi_{n}^{0}\left(\Sigma_{n}^{0}\right.$ respectively) set?

\footnotetext{
${ }^{4}$ Notice (in connection with Theorem 2.1) that $\pi[\mathcal{N}]$ is not a closed subset of $[0,1]$-for otherwise the Baire space would be compact. So the conclusion of the second assertion of Lemma 2.5 does not apply here.
} 
A positive answer to this question might be given by investigating the next question.

(2) Suppose that we are given a multi-valued function $F: X \Rightarrow Y$ for which we have that the set $F(x)$ is closed for all $x$ and $Y$ is separable. As we have proved before in case where $Y$ is exhaustible by compact sets the set of points of continuity of $F$ is $\Sigma_{3}^{0}$ and in case where $Y=\mathcal{N}$ it is possible that the latter set is not even Borel. In fact one can see that the latter is true not just for $Y=\mathcal{N}$ but also in case where $\mathcal{N}$ is topologically isomorphic with a closed subset of $Y$; (see the second assertion of Lemma 2.5 and the proof of the second assertion of Theorem 2.91). The question is what happens when $Y$ falls in neither of the previous cases i.e., $Y$ is neither exhaustible by compact sets nor it contains $\mathcal{N}$ as a closed subset. An interesting class of such examples is the class of infinite dimensional separable Banach spaces i.e., (infinite dimensional) linear normed spaces which are complete and separable under that norm. Any such space is not exhaustible by compact sets and it does not contain $\mathcal{N}$ as a closed subset. Therefore the theorems of this article provide no information in this case. It would be interesting to find the best upper bound for the complexity of the set of points of continuity of $F$ when $Y$ is an infinite dimensional separable Banach space and the set $F(x)$ is closed for all $x$.

\section{Strong Continuity.}

We continue with some results regarding the set of points of strong continuity of a multivalued function $F$. In particular we will prove the corresponding of Theorem 2.1 and Proposition 2.8. Examples which show that these results are optimum is a subject which is still under investigation. Before we proceed the author would like to express his special thanks to the anonymous referee who has provided him with very interesting remarks about strong continuity. These remarks include the observation that $F$ is strongly continuous at $x_{0}$ exactly when the multi-valued function $x \mapsto \overline{F(x)}$ (where $\overline{F(x)}$ is the topological closure of $F(x))$ is strongly continuous at $x_{0}$, which is continuity at $x_{0}$ with respect to the lower Fell topology. These remarks have motivated Proposition 3.3.

As we mentioned in the beginning, Theorem 2.1 does not require any additional hypothesis about $F$ as a subset of $X \times Y$. However the following remark suggests that this is not the case for strong continuity.

Remark 3.1. Let $A$ be a dense subset of $[0,1]$. We define the multi-valued function $F:[0,1] \Rightarrow\{0,1\}$ as follows

$$
F(x)=\{0\}, \quad \text { if } x \in A \text { and } F(x)=\{0,1\} \text { if } x \notin A,
$$

for all $x \in[0,1]$. We claim that the set of points of strong continuity of $F$ is exactly the set $A$. Let $x \in A, y \in F(x)$ and $\varepsilon>0$. Take $\delta=1>0$ and let $x^{\prime} \in(x-\delta, x+\delta)$. We have that $y=0$ and also since $0 \in F\left(x^{\prime}\right)$ we can take $y^{\prime}=0$; so $\left|y-y^{\prime}\right|=0<\varepsilon$. Now let $x \notin A$. We take $y=1 \in F(x)$ and $\varepsilon=\frac{1}{2}$. Let any $\delta>0$. Since $A$ is a dense subset of [0,1] there is some $x^{\prime} \in A$ such that $x^{\prime} \in(x-\delta, x+\delta)$. Clearly for all $y^{\prime} \in F\left(x^{\prime}\right)$ we have that $y^{\prime}=0$ and so $\left|y-y^{\prime}\right|=1>\varepsilon$. 
Since there are dense subsets of $[0,1]$ which are way above the level of analytic sets from Remark 3.1 we can see that there is no hope to obtain the corresponding of Theorem 2.1 without any additional assumptions about the complexity of the set $F$. Notice also that those assumptions about the set $F$ have to be at least as strong as the result that we want to derive. For example it is well known that there is a dense $\Pi_{3}^{0}$ set $A \subseteq[0,1]$ which is not $\Sigma_{3}^{0}$; hence by taking the multi-valued function $F$ of Remark 3.1 with respect to that set $A$ we can see that $F$ is $\Delta_{4}^{0}$ as a subset of $[0,1] \times[0,1]$ and that the set of points of strong continuity of $F$ (i.e., the set $A$ ) is not $\Sigma_{3}^{0}$. In other words if we want to result to a $\Sigma_{3}^{0}$ set we need to assume that $F$ does not go above the third level of the Borel hierarchy. The following may be regarded as the corresponding strong-continuity analogue of Theorem 2.1.

Theorem 3.2. Let $(X, p)$ and $(Y, d)$ be metric spaces with $(Y, d)$ being separable and let $F: X \Rightarrow Y$ be a multi-valued function such that $F$ is a $\Sigma_{2}^{0}$ subset of $X \times Y$.

(a) If $Y$ is compact and the set $F(x)$ is closed for all $x \in X$ then the set of points of strong continuity of $F$ is $\Pi_{2}^{0}$.

(b) If $Y$ is exhaustible by compact sets and the set $F(x)$ is closed for all $x \in X$, then the set of points of strong continuity of $F$ is $\Sigma_{3}^{0}$.

Proof. Following the proof of Theorem 2.1 we denote by $P$ the set of $x$ 's for which $F$ is strongly continuous at $x$ and also we fix a sequence $\left(y_{s}\right)_{s \in \omega}$ in $Y$ for which the set $\left\{y_{s} \mid s \in \omega\right\}$ is dense in $Y$. Let us begin with (a); we claim that

$$
\begin{aligned}
x \in P \Longleftrightarrow(\forall n)(\forall s)\left[d\left(y_{s}, F(x)\right)>\frac{1}{3(n+1)}\right. & \\
& \left.\quad \operatorname{or} \inf \left\{\sup \left\{d\left(y_{s}, F\left(x^{\prime}\right)\right) \mid x^{\prime} \in B_{p}(x, \delta)\right\} \quad \mid \delta>0\right\}<\frac{1}{n+1}\right]
\end{aligned}
$$

The proof is similar to the corresponding equivalence in the proof of Theorem 2.1. We give a brief description. For the left-to-right-hand direction notice that if $d\left(y_{s}, F(x)\right) \leq \frac{1}{3(n+1)}<$ $\frac{1}{2(n+1)}$ then there is some $y \in F(x)$ such that $d\left(y_{s}, y\right)<\frac{1}{2(n+1)}$; then proceed exactly as before. For the converse direction let $y \in F(x)$ and choose a sequence $\left(y_{s_{n}}\right)_{n \in \omega}$ such that $d\left(y_{s_{n}}, y\right)<\frac{1}{3(n+1)}$ for all $n \in \omega$. From the hypothesis we have that

$$
\inf \left\{\sup \left\{d\left(y_{s_{n}}, F\left(x^{\prime}\right)\right) \mid x^{\prime} \in B_{p}(x, \delta)\right\} \quad \mid \delta>0\right\}<\frac{1}{n+1}
$$

for all $n \in \omega$. Following the same steps as before we get the result. Now define the sets $Q_{n, s}$ and $R_{s, n}$ as follows:

$$
\begin{aligned}
& x \in Q_{n, s} \Longleftrightarrow \inf \left\{\sup \left\{d\left(y_{s}, F\left(x^{\prime}\right)\right) \mid x^{\prime} \in B_{p}(x, \delta)\right\} \mid \delta>0\right\}<\frac{1}{n+1}, \\
& x \in R_{s, n} \Longleftrightarrow d\left(y_{s}, F(x)\right) \leq \frac{1}{3(n+1)} .
\end{aligned}
$$

From $(*)$ we have that $P=\bigcap_{n, s}\left[\left(X \backslash R_{n, s}\right) \cup Q_{n, s}\right]$. We have already proved that the set $Q_{n, s}$ is open for all $n, s$, so it is enough to prove that $R_{n, s}$ is $\Sigma_{2}^{0}$ for all $n, s$. Since $F$ is $\Sigma_{2}^{0}$ we may write $F=\bigcup_{j \in \omega} F_{j}$ where $F_{j}$ is a closed subset of $X \times Y$ for all $j \in \omega$. We adopt the notation $F_{j}(x)$ for the $x$-section of $F_{j}$. Using the compactness of $F(x)$ we obtain that

$$
d\left(y_{s}, F(x)\right) \leq \frac{1}{3(n+1)} \Longleftrightarrow(\exists j) d\left(y_{s}, F_{j}(x)\right) \leq \frac{1}{3(n+1)}
$$


Define $x \in R_{n, s, j} \Longleftrightarrow d\left(y_{s}, F_{j}(x)\right) \leq \frac{1}{3(n+1)}$. We will prove that $R_{n, s, j}$ is closed for all $n, s, j$. Indeed let $\left(x_{i}\right)_{i \in \omega} \subseteq R_{n, s, j}$ be such that $x_{i} \stackrel{i \in \omega}{\longrightarrow} x$. Since $F_{j}\left(x_{i}\right)$ is compact there is some $z_{i} \in F_{j}\left(x_{i}\right)$ such that $d\left(y_{s}, z_{i}\right)=d\left(y_{s}, F_{j}\left(x_{i}\right)\right) \leq \frac{1}{3(n+1)}$ for all $i \in \omega$. From the compactness of $Y$ there is a subsequence $\left(z_{k_{i}}\right)_{i \in \omega}$ and some $z \in Y$ such that $z_{k_{i}} \stackrel{i \in \omega}{\longrightarrow} z$. Since $\left(x_{k_{i}}, z_{k_{i}}\right) \in F$ for all $i \in \omega$ and $F$ is closed we have that $(x, z) \in F$. Hence $z \in F(x)$ and so $d\left(y_{s}, F(x)\right) \leq d\left(y_{s}, z\right)=\lim _{i \in \omega} d\left(y_{s}, z_{k_{i}}\right) \leq \frac{1}{3(n+1)}$ i.e., $x \in R_{n, s, j}$.

For $(b)$, as previously we write $Y=\bigcup_{m \in \omega} K_{m}$ where $\left(K_{m}\right)_{m \in \omega}$ is a sequence of compact subsets of $Y$ such that $K_{m} \subseteq K_{m+1}^{\circ}$ for all $m \in \omega$. We claim the following modified version of $(*)$ :

$$
\begin{aligned}
x \in P \Longleftrightarrow & (\exists m)(\forall n)(\forall s)\left[d\left(y_{s}, F(x) \cap K_{m}\right)>\frac{1}{3(n+1)}\right. \\
& \left.\quad \text { or } \inf \left\{\sup \left\{d\left(y_{s}, F\left(x^{\prime}\right) \cap K_{m}\right) \mid x^{\prime} \in B_{p}(x, \delta)\right\} \quad \mid \delta>0\right\}<\frac{1}{n+1}\right]
\end{aligned}
$$

This follows from the proof of Theorem 2.1 with the modifications we described before. The relation $R_{n, s, j}^{m}$ defined by

$$
x \in R_{n, s, j}^{m} \Longleftrightarrow d\left(y_{s}, F_{j}(x) \cap K_{m}\right) \leq \frac{1}{3(n+1)}
$$

is closed; the proof is as above. (Notice that if $x \in R_{n, s, j}^{m}$ we have that $F_{j}(x) \cap K_{m} \neq \emptyset$.)

While Remark 3.1 makes it clear that the notion of strong continuity is non-metrizable in the sense of Corollary 2.7, the notions of strong continuity and metrizability are not entirely unrelated. First we put down the necessary definitions. Suppose that $Y$ is a topological space. We denote the family of all closed subsets of $Y$ by $\mathcal{F}(Y)$. The lower Fell topology on $\mathcal{F}(Y)$ is the topology generated by all sets of the form $\mathcal{A}_{U}=\{C \in \mathcal{F}(Y) \mid C \cap U \neq \emptyset\}$, where $U$ is an open subset of $Y$. We now consider the least $\sigma$-algebra $S$ on $\mathcal{F}(Y)$ containing all sets of the form $\mathcal{A}_{U}$. The pair $(\mathcal{F}(Y), S)$ is the Effros Borel space of $\mathcal{F}(Y)$. A well-known theorem states that if $Y$ is separable and metrizable by a complete distance function, (i.e., $Y$ is a Polish space) then there is a topology $\mathcal{T}$ on $\mathcal{F}(Y)$ such that: (a) the space $(\mathcal{F}(Y), \mathcal{T})$ is a Polish space and (b) the $\mathcal{T}$-Borel subsets of $\mathcal{F}(Y)$ are exactly the members of $S$, c.f. [4] Section 12.C. The Fell topology on $Y$ is the topology which has as basis the family of all sets of the form $\mathcal{W} \equiv \mathcal{W}\left(K, U_{1}, \ldots, U_{n}\right)=\left\{C \in \mathcal{F}(Y) \mid C \cap K=\emptyset \&(\forall i \leq n)\left[C \cap U_{i} \neq \emptyset\right]\right\}$, where $K$ is a compact subset of $Y$ and $U_{1}, \ldots, U_{n}$ are open subsets of $Y$. By choosing $K$ as the empty set one can see that the lower Fell topology is contained in the Fell topology. If $Y$ is a locally compact Polish space then the Fell topology is compact metrizable and its Borel space is exactly the Effros Borel space, c.f. [4] Section 12.C. Suppose that $X$ is a metric space, $Y$ is a Polish space and $F$ is a multi-valued function from $X$ to $Y$. We consider the multi-valued function $\bar{F}: X \Rightarrow Y: \bar{F}(x)=$ the topological closure of $F(x)$. We say that $F$ is pointwise-closed exactly when $F(x)$ is closed for all $x$, which is of course the same as saying that $F=\bar{F}$.

Proposition 3.3. Consider a multi-valued function $F:(X, p) \Rightarrow(Y, d)$, with $(Y, d)$ complete and separable. The following hold. 
(1) The multi-valued function $F$ is strongly continuous at $x \in X$ exactly when the multivalued function $\bar{F}$ is strongly continuous at $x$, 5 which is exactly when the function $\bar{F}: X \rightarrow \mathcal{F}(Y)$ is continuous at $x$ with respect to the lower Fell topology on $\mathcal{F}(Y)$. In particular if $\bar{F}: X \rightarrow \mathcal{F}(Y)$ is continuous at $x$ with respect to the Fell topology then $F$ is strongly continuous at $x$.

(2) Consider a Polish topology $\mathcal{T}$ on $\mathcal{F}(Y)$ such that the Borel space with respect to $\mathcal{T}$ is the Effros Borel space. If $F$ is strongly continuous at every $x \in X$, then the function $\bar{F}: X \rightarrow(\mathcal{F}(Y), \mathcal{T})$ is Borel measurable. (We are thinking of $X$ with its Borel structure.) Thus strong continuity is reduced to Borel measurability for pointwise-closed multi-valued functions with range a Polish space.

(3) If $F$ is a closed subset of $X \times Y$ and it is strongly continuous at $x \in X$ then the function $F: X \rightarrow \mathcal{F}(Y)$ is continuous at $x$ with respect to the Fell topology.

(4) Suppose $F$ is a closed subset of $X \times Y$. Then the multi-valued function $F: X \Rightarrow Y$ is strongly continuous at $x \in X$ exactly when the function $F: X \rightarrow \mathcal{F}(Y)$ is continuous at $x$ with respect to the Fell topology.

It follows that in the case of multi-valued functions with closed graph and range a locally compact Polish space, the notion of strong continuity is metrizable. In particular in any example which shows that the result of Theorem 3.2 -(b) is optimal, the set $F$ must be $\Sigma_{2}^{0}$ and not $\Pi_{1}^{0}$.

Proof.

(1) The first assertion is clear. Assume now that $\bar{F}$ is strongly continuous at $x$; let $U$ be open in $Y$ and such that $\bar{F}(x) \in \mathcal{A}_{U}$ i.e., $\bar{F}(x) \cap U \neq \emptyset$. Pick some $y \in \bar{F}(x)$ with $y \in U$ and $\varepsilon>0$ with $B_{d}(y, \varepsilon) \subseteq U$. Since $\bar{F}$ is strongly continuous at $x$, there exists some $\delta>0$ such that for all $x^{\prime} \in B_{p}(x, \delta)$ there exists some $y^{\prime} \in \bar{F}\left(x^{\prime}\right)$ such that $y^{\prime} \in B_{d}(y, \varepsilon)$. Hence $\bar{F}\left(x^{\prime}\right) \cap U \neq \emptyset$ for all $x^{\prime} \in B_{p}(x, \delta)$. Conversely assume that $\bar{F}$ is continuous at $x$ with respect to the lower Fell topology; let $y \in \bar{F}(x)$ and $\varepsilon>0$. We consider the open neighborhood $\left\{C \in \mathcal{F}(Y) \mid C \cap B_{d}(y, \varepsilon) \neq \emptyset\right\}$ of $\bar{F}(x)$. There exists some $\delta>0$ such that for all $x^{\prime} \in B_{p}(x, \delta)$ we have that $\bar{F}\left(x^{\prime}\right) \cap B_{d}(y, \varepsilon) \neq \emptyset$. This means that there exists some $\delta>0$ such that for all $x^{\prime} \in B_{p}(x, \delta)$ there exists some $y^{\prime} \in \bar{F}\left(x^{\prime}\right)$ with $d\left(y, y^{\prime}\right)<\varepsilon$.

The last assertion of 1 . follows from the fact that the lower Fell topology is contained in the Fell topology.

(2) This is an immediate consequence of 1 . and the comments about the Effros Borel space proceeding Proposition 3.3 .

(3) This is similar to the proof of 1 . Suppose that

$$
\mathcal{W}=\left\{C \in \mathcal{F}(Y) \mid C \cap K=\emptyset \&(\forall i \leq n)\left[C \cap U_{i} \neq \emptyset\right]\right\}
$$

is a basic neighborhood of $F(x)$ with respect to the Fell topology. Choose $y_{i} \in F(x) \cap U_{i}$ and $\varepsilon_{i}>0$ with $B_{d}\left(y_{i}, \varepsilon_{i}\right) \subseteq U_{i}$ for all $i=1, \ldots, n$. From our hypothesis there is some $\delta_{0}>0$ such that for all $x^{\prime} \in B_{p}\left(x, \delta_{0}\right)$ we have that $F\left(x^{\prime}\right) \cap B_{d}\left(y_{i}, \varepsilon_{i}\right) \neq \emptyset$ for all $i=1, \ldots, n$.

Now we claim that there is some $0<\delta<\delta_{0}$ such that for all $x^{\prime} \in B_{p}(x, \delta)$ we have that $F\left(x^{\prime}\right) \cap K=\emptyset$. Indeed if this is not the case, then there are sequences $\left(x_{n}\right)_{n \in \omega}$ and $\left(z_{n}\right)_{n \in \omega}$ such that $x_{n} \rightarrow x$ and $z_{n} \in F\left(x_{n}\right) \cap K$ for all $n \in \omega$. From the compactness of $K$ there is a subsequence $\left(z_{k_{n}}\right)_{n \in \omega}$ which converges to some $z \in K$.

\footnotetext{
${ }^{5}$ Unfortunately this observation does not seem to allow us to drop the hypothesis " $F(x)$ is closed" in Theorem 3.2
} 
Therefore $\left(x_{k_{n}}, z_{k_{n}}\right) \rightarrow(x, z)$. Since $\left(x_{k_{n}}, z_{k_{n}}\right) \in F$ for all $n \in \omega$ and $F$ is closed it follows that $z \in F(x)$. Thus $F(x) \cap K \neq \emptyset$ contradicting that $\mathcal{W}$ is a neighborhood of $F(x)$.

It follows that there is some $\delta>0$ such that for all $x^{\prime} \in B_{p}(x, \delta)$ we have that $F\left(x^{\prime}\right) \in \mathcal{W}$.

(4) The first assertion is clear from 1. and 3. The second assertion follows from the fact that when $Y$ is Polish and locally compact the Fell topology is compact metrizable. The assertion about Theorem 3.2 follows from the fact that every space which is exhaustible by compact sets is locally compact.

We continue with the corresponding of Proposition 2.8,

Proposition 3.4. Let $(X, p)$ and $(Y, d)$ be complete and separable metric spaces and let $F: X \Rightarrow Y$ be a multi-valued function such that the set $F \subseteq X \times Y$ is analytic. Then the set of points of strong continuity of $F$ is co-analytic.

Proof. As in the proof of Proposition 2.8 for all $n \in \omega$ we define the set $A_{n} \subseteq X \times Y$ as follows

$$
(x, y) \in A_{n} \Longleftrightarrow \inf \left\{\sup \left\{d\left(y, F\left(x^{\prime}\right)\right) \mid x^{\prime} \in B_{p}(x, \delta)\right\} \quad \mid \delta>0\right\}<\frac{1}{n+1} .
$$

As before the set $A_{n}$ is open for all $n \in \omega$. Let $P_{s}$ be the set of points of strong continuity of $F$. It follows that

$$
\begin{aligned}
x \in P_{s} & \Longleftrightarrow(\forall y \in F(x))(\forall n)\left[(x, y) \in A_{n}\right] \\
& \Longleftrightarrow(\forall y)\left[(x, y) \notin F \text { or }(\forall n)\left[(x, y) \in A_{n}\right]\right] .
\end{aligned}
$$

So

$$
x \notin P_{s} \Longleftrightarrow(\exists y)\left[(x, y) \in F \&(\exists n)\left[(x, y) \notin A_{n}\right]\right] .
$$

Since $F$ is analytic and since every set $A_{n}$ is open we obtain that the complement of $P_{s}$ is analytic i.e., the set $P_{s}$ is co-analytic.

We conclude this article with some remarks which concern all previous results. The author would like to thank the anonymous referee for raising the questions stated below.

Remark 3.5. All results above are in the context of classical descriptive set theory. One could ask whether the corresponding results are also true in the context of effective descriptive set theory. In the latter context one deals with the notion of a recursive function $f: \omega^{k} \rightarrow \omega^{m}$ and of a recursive subset of $\omega^{k}$. We assume that our given metric space $(X, d)$ is complete, separable and that there is a countable dense sequence $\left\{r_{i} \mid i \in \omega\right\}$ such that the relations $d\left(r_{i}, r_{j}\right)<q, d\left(r_{i}, r_{j}\right) \leq q$ for $i, j \in \omega$ and $q \in \mathbb{Q}^{+}$, are recursive. (An example of such space is $\mathbb{R}$ with $\left\{r_{i} \mid i \in \omega\right\}=\mathbb{Q}$.) One takes then the family $\{N(X, s) \mid s \in \omega\}$ of all open balls with centers from the set $\left\{r_{i} \mid i \in \omega\right\}$ and rational radii and defines the class of semirecursive sets or "effectively open" sets as the sets which are recursive unions of sets of the form $N(X, s)$. The analogous notions go through the whole hierarchy of Borel and analytical sets i.e., one constructs the family of effectively closed, effectively $G_{\delta}$, effectively analytic sets and so on. The latter classes of sets are also called lightface classes. The usual inclusion properties hold also for the lightface classes. For example every effectively closed set is effectively $G_{\delta}$. We should point out that there are only countably many subsets of a fixed space $X$ which belong to a specific lightface class. Also all singletons $\{q\}$ with $q \in \mathbb{Q}$ belong to every one of the lightface classes mentioned above except from the one 
of semi-recursive sets. The reader can refer to [5] for a detailed exposition of this theory. One natural question which arises is if the results which are presented in this article hold in the context of effective descriptive set theory. For example: if $F: \mathbb{R} \Rightarrow \mathbb{R}$ is a bounded multi-valued function such that the set $F(x)$ is effectively closed, is it true that the set of points of continuity of $F$ is effectively $G_{\delta}$ ? As the next proposition shows the answer to this question is negative even if $F$ is a single-valued function.

Let us say that a family of sets $\Gamma$ is closed under negation if whenever $A \subseteq X$ is in $\Gamma$ then $X \backslash A$ is in $\Gamma$ as well.

Proposition 3.6. Suppose that $\Gamma$ is a class of sets which is closed under negation and the family

$\{A \subseteq \mathbb{R} \mid A \in \Gamma\}$ is countable. Then there is a function $f: \mathbb{R} \rightarrow\left\{\frac{1}{n+1} \mid n \in \omega\right\} \cup\{0\}$ such that the set of points of continuity of $f$ is not a member of $\Gamma$. In particular (by choosing $\Gamma$ as the lightface $\Delta_{2}^{1}$ class) there is a function $f: \mathbb{R} \rightarrow[0,1]$ such that the singleton $\{f(x)\}$ is effectively closed for all $x \in \mathbb{R}$ but the set of points of continuity of $f$ is neither effectively analytic nor effectively co-analytic.

Proof. Since $\Gamma$ restricted on the subsets of $\mathbb{R}$ is countable there is some $A \subseteq \mathbb{R}$ which is infinite countable set and not a member of $\Gamma$. Write $A=\left\{x_{n} \mid n \in \omega\right\}$ with $x_{n} \neq x_{m}$ for $n \neq m$ and define $f: \mathbb{R} \rightarrow \mathbb{R}$ as follows: $f(x)=0$ if $x \notin A$ and $f(x)=\frac{1}{n+1}$ if $x=x_{n}$.

We claim that the set of points of continuity of $f$ is $\mathbb{R} \backslash A$. Since $A$ is not a member of $\Gamma$ and the class $\Gamma$ is closed under negation we also have that the set $\mathbb{R} \backslash A$ is not a member of $\Gamma$. So if we prove our claim we are done.

Assume that $x \in A$ and $x=x_{n}$. Choose $\varepsilon=\frac{1}{2(n+1)}>0$ and let any $\delta>0$. The interval $(x-\delta, x+\delta)$ is uncountable so it contains some $y$ which is not a member of $A$. It follows that $f(y)=0$ and so $|f(y)-f(x)|=f(x)=\frac{1}{n+1}>\varepsilon$. This shows that no point of $A$ is a point of continuity of $f$. Now assume that $x \notin A$ and let arbitrary $\varepsilon>0$. Choose $n \in \omega$ such that $\frac{1}{n+1}<\varepsilon$ and define

$$
\delta=\min \left\{\left|x-x_{i}\right| \quad \mid i=0, \ldots, n\right\} .
$$

Since $x \notin A$ we have that $\delta>0$. Now let any $y \in(x-\delta, x+\delta)$; if $y \in A$ then $y=x_{m}$ for some $m>n$, so $|f(y)-f(x)|=f(y)=\frac{1}{m+1}<\frac{1}{n+1}<\varepsilon$. If $y \notin A$ then clearly $|f(y)-f(x)|=0<\varepsilon$. Therefore the function $f$ is continuous at $x$.

Question 2. In case we take $\Gamma$ to be the lightface $\Delta_{n}^{0}$ class for some small $n \in \omega$, it would be interesting to see whether one can construct a function $f$ which satisfies the first conclusion of the previous proposition and has the additional property that the graph of $f$ belongs to $\Gamma$.

\section{REFERENCES}

[1] Z. Adamowicz, A generalization of the Shoenfield theorem on $\Sigma_{2}^{1}$ sets, Continuous relations and generalized $G_{\delta}$-sets, Fund.Math., 123, pp. 81-107 (1984).

[2] Vasco Brattka, Peter Hertling, Continuity and Computability of Relations, Informatik Berichte 164 FernUniversität in Hagen (1994).

[3] G. Choquet, Convergences, Annales de l'Université de Grenoble 23, pp. 55-112, (1947-1948).

[4] A.S. Kechris, Classical Descriptive Set Theory, Graduate Texts in Mathematics, vol. 156, SpringerVerlag, 1995.

[5] Y.N. Moschovakis, Descriptive Set Theory, Studies in Logic and the Foundations of Mathematics, 100, North-Holland Publishing Co., 1980. 
[6] M. Ziegler, Real Computation with Least Discrete Advice: A Complexity Theory of Nonuniform Computability with Applications to Effective Linear Algebra, to appear in Ann. Pure Appl. Logic.

This work is licensed under the Creative Commons Attribution-NoDerivs License. To view a copy of this license, visit http://creativecommons.org/licenses/by-nd/2.0/ or send a letter to Creative Commons, 171 Second St, Suite 300, San Francisco, CA 94105, USA, or Eisenacher Strasse 2, 10777 Berlin, Germany 